OSU-HEP-10-04

June 2010

\title{
Semidirect Product Groups, Vacuum Alignment and Tribimaximal Neutrino Mixing
}

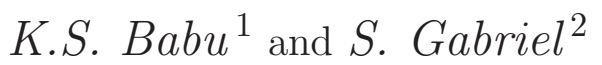 \\ Department of Physics, Oklahoma State University, Stillwater, OK 74078, USA
}

\begin{abstract}
The neutrino oscillation data are in very good agreement with the tribimaximal mixing pattern: $\sin ^{2} \theta_{23}=1 / 2, \sin ^{2} \theta_{12}=1 / 3$, and $\sin ^{2} \theta_{13}=0$. Attempts to generate this pattern based on finite family symmetry groups typically assume that the family symmetry is broken to different subgroups in the charged lepton and the neutrino mass matrices. This leads to a technical problem, where the cross-couplings between the Higgs fields responsible for the two symmetry breaking chains force their vacuum expectation values to align, upsetting the desired breaking pattern. Here, we present a class of models based on the semidirect product group $\left(S_{3}\right)^{4} \rtimes A_{4}$, where the lepton families belong to representations which are not faithful. In effect, the Higgs sector knows about the full symmetry while the lepton sector knows only about the $A_{4}$ factor group. This can solve the alignment problem without altering the desired properties of the family symmetry. Inclusion of quarks into the framework is straightforward, and leads to small and arbitrary CKM mixing angles. Supersymmetry is not essential for our proposal, but the model presented is easily supersymmetrized, in which case the same family symmetry solves the SUSY flavor problem.
\end{abstract}

\footnotetext{
${ }^{1}$ Email: babu@okstate.edu

${ }^{2}$ svengab@msn.com
} 


\section{Introduction}

The neutrino mixing angles are now known to some accuracy [1, 2]. A global analysis of data shows (in the standard parametrization of the lepton mixing matrix) [1]:

$$
\sin ^{2} \theta_{23}=0.50_{-0.06}^{+0.07}, \quad \sin ^{2} \theta_{12}=0.304_{-0.016}^{+0.022}, \quad \sin ^{2} \theta_{13}<0.056
$$

where the error bars correspond to $1 \sigma$ variations and the upper limit is at the $3 \sigma$ level. These values are in good agreement with the tribimaximal mixing pattern given by the leptonic mixing matrix $[3,4]$

$$
U_{M N S}=\left(\begin{array}{ccc}
\sqrt{\frac{2}{3}} & \frac{1}{\sqrt{3}} & 0 \\
-\frac{1}{\sqrt{6}} & \frac{1}{\sqrt{3}} & -\frac{1}{\sqrt{2}} \\
-\frac{1}{\sqrt{6}} & \frac{1}{\sqrt{3}} & \frac{1}{\sqrt{2}}
\end{array}\right) P
$$

where $P$ is a diagonal phase matrix. This matrix corresponds to geometric values of the mixing parameters

$$
\sin ^{2} \theta_{23}=1 / 2, \quad \sin ^{2} \theta_{12}=1 / 3, \quad \sin ^{2} \theta_{13}=0
$$

which are all in good agreement presently with the experimental values quoted in (11).

It has been known for some time that the tribimaximal mixing pattern of (2) can be obtained using finite family symmetry groups such as $A_{4}$ [5, 6, 7, 8, 9, 10, 11, 12, 13, 14, and others [15]. In the class of models based on $A_{4}$, the standard model (SM) lepton doublets are assigned to triplet representation of $A_{4}$, and the $A_{4}$ symmetry is broken to a $Z_{2}$ subgroup in the neutrino sector by a triplet Higgs $\chi$, with the vacuum expectation value (VEV) structure $\langle\chi\rangle \propto(0,1,0)$ or some permutation thereof, and to a $Z_{3}$ subgroup in the charged lepton sector by a triplet Higgs $\phi$, with the VEV structure $\langle\phi\rangle \propto(1,1,1)$. However, there is a technical problem with this, in that couplings between the Higgs fields $\chi$ and $\phi$ will force their VEVs to align, upsetting the desired breaking pattern. Several approaches have been adopted in the literature to overcome this vacuum alignment problem. One can introduce more complicated symmetries, and assume that some symmetries are softly broken. Alternatively, one can assume that the symmetry is only approximate, and that non-renormalizable operators are present in the Lagrangian. Supersymmetry helps, since the alignment problem exists in this context only at the cubic level in the superpotential. Care must be taken to ensure that there are no flat directions - a frequent occurrence in SUSY models. The existence of extra dimensions is sometimes assumed, where the two Higgs fields can be separated, thus circumventing the alignment problem. While all of these approaches are interesting, in our opinion, a compelling scenario has not emerged, arising from renormalizable models with exact symmetries realized in the Higgs phase.

In this paper we propose a new approach to address the vacuum alignment problem using semidirect product groups of a type usually not considered, which provides a technically complete picture for tribimaximal lepton mixing. Supersymmetry is not essential, although the 
idea presented is compatible with SUSY. The symmetries of the model are assumed to be spontaneously (and not softly) broken. Non-renormalizable operators are not necessary, although their existence does not upset the desired VEV structure.

We consider models where the SM lepton families belong to representations of a finite symmetry which are not faithful (that is, not every member of the group is represented by a distinct transformation). In effect, the Higgs sector knows about the full symmetry while the lepton sector does not. Our framework is a renormalizable extension of the standard model with an additional finite family symmetry $G \times Z_{2}$, with $G=S_{3}^{4} \rtimes A_{4}$. The rationale for the choice of this group is elaborated in Sec. II. Here we note briefly that $S_{3}$ is the smallest nonAbelian group, and that four such factors would allow one to evenly permute them, which is the symmetry operation of $A_{4}$. This enables us to form a non-trivial semidirect product group, which has all the desired properties for solving the vacuum alignment problem. This group contains $S_{3}^{4}$ as an invariant subgroup, so that it will have representations corresponding to the factor group $G / S_{3}^{4} \sim A_{4}$. The standard model leptons can then be assigned to representations of $A_{4}$, as has been done in the models of Ref. [5, 6, 7, 8, 9, 10, 11, 12, 13, 14]. Neutrino masses are generated by a Higgs field $\phi$, belonging to a $Z_{2}$ even 16-dimensional representation of $G$, while charged-lepton masses are generated by a Higgs field $\chi$, belonging to a $Z_{2}$ odd triplet of $A_{4}$. The $Z_{2}$ symmetry prevents cubic and non-trivial quartic interactions between $\phi$ and $\chi$. In this way, the alignment problem is solved without altering the desired properties of the family symmetry. Inclusion of the quark sector into the framework is straightforward, and leads to arbitrary and small CKM mixing angles. The model can readily be supersymmetrized, as discussed in Sec. VI. In this case the same family symmetry can solve the SUSY flavor problem.

In Sec. II we present our model based on $\left(S_{3}^{4} \rtimes A_{4}\right) \times Z_{2}$ family symmetry. In Sec. III the Higgs potential of the model is analyzed, where it is established that the model has no vacuum alignment problem. Here we also assert, by computing the scalar boson masses, that the symmetry breaking is complete, without leading to any pseudo-Goldston bosons. Sec. IV discusses the lepton mass matrices, and the resulting tribimaximal lepton mixing matrix. In Sec. $\mathrm{V}$ we discuss briefly the inclusion of quarks in the framework. Sec. VI discusses supersymmetrization of the model. In Sec. VII we conclude. In four Appendices we provide various technical details: the generators of the group in matrix form, invariants of the group we need, and the calculations of the light charged lepton and neutrino mass matrices.

\section{Model of $\left(S_{3}^{4} \rtimes A_{4}\right) \times Z_{2}$ family symmetry}

The gauge symmetry of our model is $S U(3)_{c} \times S U(2)_{L} \times U(1)_{Y}$, and we work in its minimal non-supersymmetric version. We extend the gauge symmetry by the finite family symmetry $G \times Z_{2}$, where $G$ has the semi-direct product 3 structure $\left(S_{3} \times S_{3} \times S_{3} \times S_{3}\right) \rtimes A_{4}$. The finite

\footnotetext{
${ }^{3}$ The semi-direct product, $N \rtimes H$, contains $N$ and $H$ as subgroups and obeys $h n h^{-1} \in N$ for all $n \in N$ and $h \in H$ [16. Thus, $N$ is an invariant subgroup. The number of elements in the group, denoted by $|N \rtimes H|$, is
} 
symmetry is broken at a scale $M_{*}$ which is assumed to be large compared to the EW scale. $M_{*}$ may be identified as the grand unification scale, or it may be an intermediate scale.

\subsection{Symmetry group}

The group $A_{4}$ can be described using two generators obeying the relations,

$$
X^{2}=Y^{3}=E, \quad X Y X=Y^{2} X Y^{2}
$$

where $E$ is the identity. The irreducible representations of $A_{4}$ are one real singlet, two complex singlets, and one real triplet. Table 1 gives $X$ and $Y$ in each of these representations. The $S_{3}$ generators, $A_{i}$ and $B_{i}$ obey

$$
A_{i}^{3}=B_{i}^{2}=E, \quad B_{i} A_{i} B_{i}^{-1}=A_{i}^{-1} .
$$

The irreducible representations of $S_{3}$ are two real singlets and one real doublet. The generators in each of these representations can also be found in Table 1. The remaining relations defining the full symmetry are

$$
\begin{gathered}
X A_{1} X^{-1}=A_{2}, \quad X A_{2} X^{-1}=A_{1}, \quad X A_{3} X^{-1}=A_{4}, \quad X A_{4} X^{-1}=A_{3}, \\
X B_{1} X^{-1}=B_{2}, \quad X B_{2} X^{-1}=B_{1}, \quad X B_{3} X^{-1}=B_{4}, \quad X B_{4} X^{-1}=B_{3}, \\
Y A_{1} Y^{-1}=A_{1}, \quad Y A_{2} Y^{-1}=A_{3}, \quad Y A_{3} Y^{-1}=A_{4}, \quad Y A_{4} Y^{-1}=A_{2}, \\
Y B_{1} Y^{-1}=B_{1}, \quad Y B_{2} Y^{-1}=B_{3}, \quad Y B_{3} Y^{-1}=B_{4}, \quad Y B_{4} Y^{-1}=B_{2} .
\end{gathered}
$$

It's easy to see that if $A_{1}, A_{2}, A_{3}, A_{4}, B_{1}, B_{2}, B_{3}$, and $B_{4}$ are all represented by the identity matrix, then (5), (6), and (7) are trivially satisfied, leaving only (4) to be checked. But finding representations obeying (4) corresponds to finding the representations of $A_{4}$, which are given in Table 1. These representations are used for SM leptons. The character table of $S_{3}^{4} \rtimes A_{4}$ is given in Table 2 for completeness. The first four representations listed in Table 2 correspond to the representations of $A_{4}$.

$|N||H|$. The semi-direct product exists when $H$ has a factor group which is a subgroup of the automorphism group of $N$. 


\begin{tabular}{|c|c|c|c|}
\hline & $\mathrm{X}$ & $\mathrm{Y}$ \\
\hline 1 & & 1 & 1 \\
$1^{\prime}$ & & & \\
$1^{\prime \prime}$ & & \\
& 1 & \\
3 & & $\omega^{2}$ \\
& & $\left(\begin{array}{ccc}-1 & 0 & 0 \\
0 & 1 & 0 \\
0 & 0 & -1\end{array}\right)$ & $\left(\begin{array}{lll}0 & 0 & 1 \\
1 & 0 & 0 \\
0 & 1 & 0\end{array}\right)$ \\
\hline
\end{tabular}

\begin{tabular}{|c|c|c|}
\hline & $A_{i}$ & $B_{i}$ \\
\hline 1 & 1 & 1 \\
$1^{\prime}$ & 1 \\
2 & $\left(\begin{array}{cc}\omega & 0 \\
0 & \omega^{2}\end{array}\right)$ & $\left(\begin{array}{ll}0 & 1 \\
1 & 0\end{array}\right)$ \\
\hline
\end{tabular}

Table 1: The left panel shows the matrices representing the generators in each irrep. of $A_{4}$, in a certain basis. The right panel shows the same for $S_{3}$. Here $\omega=e^{2 i \pi / 3}$. 


\begin{tabular}{|c|c|c|c|c|c|c|c|c|c|c|c|c|c|c|c|c|c|c|}
\hline & 1 & 8 & 24 & 16 & 12 & 54 & 108 & 81 & 144 & 216 & 96 & 216 & 216 & 108 & 432 & 648 & 1296 & $\ldots$ \\
\hline 1 & 1 & 1 & 1 & 1 & 1 & 1 & 1 & 1 & 1 & 1 & 1 & 1 & 1 & 1 & 1 & 1 & 1 & $\ldots$ \\
\hline $1^{\prime}$ & 1 & 1 & 1 & 1 & 1 & 1 & 1 & 1 & 1 & 1 & 1 & 1 & 1 & 1 & 1 & 1 & 1 & $\ldots$ \\
\hline $1^{\prime \prime}$ & 1 & 1 & 1 & 1 & 1 & 1 & 1 & 1 & 1 & 1 & 1 & 1 & 1 & 1 & 1 & 1 & 1 & $\ldots$ \\
\hline 3 & 3 & 3 & 3 & 3 & 3 & 3 & 3 & 3 & 3 & 3 & 3 & 3 & 3 & -1 & -1 & -1 & -1 & $\ldots$ \\
\hline$\widetilde{1}$ & 1 & 1 & 1 & 1 & -1 & 1 & -1 & -1 & -1 & 1 & -1 & 1 & -1 & 1 & 1 & -1 & -1 & $\ldots$ \\
\hline$\widetilde{1}^{\prime}$ & 1 & 1 & 1 & 1 & -1 & 1 & -1 & -1 & -1 & 1 & -1 & 1 & -1 & 1 & 1 & -1 & -1 & $\ldots$ \\
\hline$\widetilde{1}_{\sim}^{\prime \prime}$ & 1 & 1 & 1 & 1 & -1 & 1 & -1 & -1 & -1 & 1 & -1 & 1 & -1 & 1 & 1 & -1 & -1 & $\ldots$ \\
\hline$\widetilde{3}$ & 3 & 3 & 3 & 3 & -3 & 3 & -3 & -3 & -3 & 3 & -3 & 3 & -3 & -1 & -1 & 1 & 1 & $\ldots$ \\
\hline 4 & 4 & 4 & 4 & 4 & 2 & 0 & -2 & -4 & 2 & 0 & 2 & 0 & -2 & 0 & 0 & 0 & 0 & $\ldots$ \\
\hline $4^{\prime}$ & 4 & 4 & 4 & 4 & 2 & 0 & -2 & -4 & 2 & 0 & 2 & 0 & -2 & 0 & 0 & 0 & 0 & $\ldots$ \\
\hline $4^{\prime \prime}$ & 4 & 4 & 4 & 4 & 2 & 0 & -2 & -4 & 2 & 0 & 2 & 0 & -2 & 0 & 0 & 0 & 0 & $\ldots$ \\
\hline$\widetilde{4}$ & 4 & 4 & 4 & 4 & -2 & 0 & 2 & -4 & -2 & 0 & -2 & 0 & 2 & 0 & 0 & 0 & 0 & $\ldots$ \\
\hline$\widetilde{\widetilde{4}}^{\prime}$ & 4 & 4 & 4 & 4 & -2 & 0 & 2 & -4 & -2 & 0 & -2 & 0 & 2 & 0 & 0 & 0 & 0 & $\ldots$ \\
\hline$\widetilde{4}^{\prime \prime}$ & 4 & 4 & 4 & 4 & -2 & 0 & 2 & -2 & -2 & 0 & -2 & 0 & 2 & 0 & 0 & 0 & 0 & $\ldots$ \\
\hline 6 & 6 & 6 & 6 & 6 & 0 & -2 & 0 & 6 & 0 & -2 & 0 & -2 & 0 & 2 & 2 & 0 & 0 & $\ldots$ \\
\hline $6^{\prime}$ & 6 & 6 & 6 & 6 & 0 & -2 & 0 & 0 & 0 & -2 & 0 & -2 & 0 & -2 & -2 & 0 & 0 & $\ldots$ \\
\hline 8 & 8 & 5 & -1 & -4 & 6 & 4 & 2 & 3 & 0 & 1 & -3 & -2 & -1 & 0 & 0 & 0 & 0 & $\ldots$ \\
\hline $8^{\prime}$ & 8 & 5 & -1 & -4 & 6 & 4 & 2 & 3 & 0 & 1 & -3 & -2 & -1 & 0 & 0 & 0 & 0 & $\ldots$ \\
\hline $8^{\prime \prime}$ & 8 & 5 & -1 & -4 & 6 & 4 & 2 & 3 & 0 & 1 & -3 & -2 & -1 & 0 & 0 & 0 & 0 & $\ldots$ \\
\hline$\widetilde{8}$ & 8 & 5 & -1 & -4 & -6 & 4 & -2 & -3 & 0 & 1 & 3 & -2 & 1 & 0 & 0 & 0 & 0 & $\ldots$ \\
\hline$\widetilde{8}^{\prime}$ & 8 & 5 & -1 & -4 & -6 & 4 & -2 & -3 & 0 & 1 & 3 & -2 & 1 & 0 & 0 & 0 & 0 & $\ldots$ \\
\hline$\widetilde{8}^{\prime \prime}$ & 8 & 5 & -1 & -4 & -6 & 4 & -2 & -3 & 0 & 1 & 3 & -2 & 1 & 0 & 0 & 0 & 0 & $\ldots$ \\
\hline 16 & 16 & -8 & -2 & 1 & 0 & 0 & 0 & 0 & 0 & 0 & 0 & 0 & 0 & -2 & 1 & 0 & 0 & $\ldots$ \\
\hline $16^{\prime}$ & 16 & -8 & -2 & 1 & 0 & 0 & 0 & 0 & 0 & 0 & 0 & 0 & 0 & -2 & 1 & 0 & 0 & $\ldots$ \\
\hline $16^{\prime \prime}$ & 16 & -8 & -2 & 1 & 0 & 0 & 0 & 0 & 0 & 0 & 0 & 0 & 0 & -2 & 1 & 0 & 0 & $\ldots$ \\
\hline 24 & 24 & 6 & -3 & 6 & 12 & 4 & 0 & 0 & -3 & -2 & 3 & 1 & 0 & 1 & -2 & 2 & -1 & $\ldots$ \\
\hline $24^{\prime}$ & 24 & 6 & -3 & 6 & 12 & 4 & 0 & 0 & -3 & -2 & 3 & 1 & 0 & -4 & 2 & -2 & 1 & $\ldots$ \\
\hline$\widetilde{24}$ & 24 & 6 & -3 & 6 & -12 & 4 & 0 & 0 & 3 & -2 & -3 & 1 & 0 & 4 & -2 & -2 & 1 & $\ldots$ \\
\hline$\widetilde{24}^{\prime}$ & 24 & 6 & -3 & 6 & -12 & 4 & 0 & 0 & 3 & -2 & -3 & 1 & 0 & -4 & 2 & 2 & -1 & $\ldots$ \\
\hline $24^{\prime \prime}$ & 24 & 15 & -3 & -12 & 6 & -4 & -6 & 3 & 0 & -1 & -3 & 2 & 3 & 0 & 0 & 0 & 0 & $\ldots$ \\
\hline$\widetilde{24}^{\prime \prime}$ & 24 & 15 & -3 & -12 & -6 & -4 & 6 & -3 & 0 & -1 & 3 & 2 & -3 & 0 & 0 & 0 & 0 & $\ldots$ \\
\hline 32 & 32 & -4 & -4 & -4 & 8 & 0 & 0 & -4 & 2 & 0 & -1 & 0 & 0 & 0 & 0 & 0 & 0 & $\ldots$ \\
\hline $32^{\prime}$ & 32 & -4 & -4 & -4 & 8 & 0 & 0 & -4 & 2 & 0 & -1 & 0 & 0 & 0 & 0 & 0 & 0 & $\ldots$ \\
\hline $32^{\prime \prime}$ & 32 & -4 & -4 & -4 & 8 & 0 & 0 & -4 & 2 & 0 & -1 & 0 & 0 & 0 & 0 & 0 & 0 & $\ldots$ \\
\hline 32 & 32 & -4 & -4 & -4 & -8 & 0 & 0 & 4 & -2 & 0 & 1 & 0 & 0 & 0 & 0 & 0 & 0 & $\ldots$ \\
\hline$\widetilde{32}^{\prime}$ & 32 & -4 & -4 & -4 & -8 & 0 & 0 & 0 & -2 & 0 & 1 & 0 & 0 & 0 & 0 & 0 & 0 & $\ldots$ \\
\hline$\widetilde{32}^{\prime \prime}$ & 32 & -4 & -4 & -4 & -8 & 0 & 0 & 4 & -2 & 0 & 1 & 0 & 0 & 0 & 0 & 0 & 0 & $\ldots$ \\
\hline 48 & 48 & -24 & 12 & 3 & 0 & 0 & 0 & 0 & 0 & 0 & 0 & 0 & 0 & -4 & -1 & 0 & 0 & $\ldots$ \\
\hline 48 & 48 & 12 & -6 & 12 & 0 & \begin{tabular}{l|l}
-8 & \\
\end{tabular} & 0 & 0 & 0 & 4 & 0 & -2 & 0 & 0 & 0 & 0 & 0 & $\ldots$ \\
\hline & 972 & 144 & 288 & 576 & 432 & 1296 & 864 & 288 & 432 & 864 & 144 & 288 & 576 & 432 & 1296 & 864 & 288 & 432 \\
\hline 1 & 1 & 1 & 1 & 1 & 1 & 1 & 1 & 1 & 1 & 1 & 1 & 1 & 1 & 1 & 1 & 1 & 1 & 1 \\
\hline $1^{\prime}$ & 1 & $\omega$ & $\omega$ & $\omega$ & $\omega$ & $\omega$ & $\omega$ & $\omega$ & $\omega$ & $\omega$ & $\omega^{2}$ & $\omega^{2}$ & $\omega^{2}$ & $\omega^{2}$ & $\omega^{2}$ & $\omega^{2}$ & $\omega^{2}$ & $\omega^{2}$ \\
\hline $1^{\prime \prime}$ & 1 & $\omega^{2}$ & $\omega^{2}$ & $\omega^{2}$ & $\omega^{2}$ & $\omega^{2}$ & $\omega^{2}$ & $\omega^{2}$ & $\omega^{2}$ & $\omega^{2}$ & $\omega$ & $\omega$ & $\omega$ & $\omega$ & $\omega$ & $\omega$ & $\omega$ & $\omega$ \\
\hline 3 & -1 & 0 & 0 & 0 & 0 & 0 & 0 & 0 & 0 & 0 & 0 & 0 & 0 & 0 & 0 & 0 & 0 & 0 \\
\hline$\widetilde{1}$ & 1 & 1 & 1 & 1 & -1 & 1 & -1 & 1 & -1 & -1 & 1 & 1 & 1 & -1 & 1 & -1 & 1 & -1 \\
\hline$\widetilde{1}^{\prime}$ & 1 & $\omega$ & $\omega$ & $\omega$ & $-\omega$ & $\omega$ & $-\omega$ & $\omega$ & $-\omega$ & $-\omega$ & $\omega^{2}$ & $\omega^{2}$ & $\omega^{2}$ & $-\omega^{2}$ & $\omega^{2}$ & $-\omega^{2}$ & $\omega^{2}$ & $-\omega^{2}$ \\
\hline$\widetilde{1}^{\prime \prime}$ & 1 & $\omega^{2}$ & $\omega^{2}$ & $\omega^{2}$ & $-\omega^{2}$ & $\omega^{2}$ & $-\omega^{2}$ & $\omega^{2}$ & $-\omega^{2}$ & $-\omega^{2}$ & $\omega$ & $\omega$ & $\omega$ & $-\omega$ & $\omega$ & $-\omega$ & $\omega$ & $-\omega$ \\
\hline$\widetilde{3}$ & -1 & 0 & 0 & 0 & 0 & 0 & 0 & 0 & 0 & 0 & 0 & 0 & 0 & 0 & 0 & 0 & 0 & 0 \\
\hline 4 & 0 & 1 & 1 & 1 & -1 & -1 & 1 & 1 & 1 & -1 & 1 & 1 & 1 & -1 & -1 & 1 & 1 & 1 \\
\hline $4^{\prime}$ & 0 & $\omega$ & $\omega$ & $\omega$ & $-\omega$ & $-\omega$ & $\omega$ & $\omega$ & $\omega$ & $-\omega$ & $\omega^{2}$ & $\omega^{2}$ & $\omega^{2}$ & $-\omega^{2}$ & $-\omega^{2}$ & $\omega^{2}$ & $\omega^{2}$ & $\omega^{2}$ \\
\hline $4^{\prime \prime}$ & 0 & $\omega^{2}$ & $\omega^{2}$ & $\omega^{2}$ & $-\omega^{2}$ & $-\omega^{2}$ & $\omega^{2}$ & $\omega^{2}$ & $\omega^{2}$ & $-\omega^{2}$ & $\omega$ & $\omega$ & $\omega$ & $-\omega$ & $-\omega$ & $\omega$ & $\omega$ & $\omega$ \\
\hline$\widetilde{4}$ & 0 & 1 & 1 & 1 & 1 & -1 & -1 & 1 & -1 & 1 & 1 & 1 & 1 & 1 & -1 & -1 & 1 & -1 \\
\hline$\tilde{4}^{\prime}$ & 0 & $\omega$ & $\omega$ & $\omega$ & $\omega$ & $-\omega$ & $-\omega$ & $\omega$ & $-\omega$ & $\omega$ & $\omega^{2}$ & $\omega^{2}$ & $\omega^{2}$ & $\omega^{2}$ & $-\omega^{2}$ & $-\omega^{2}$ & $\omega^{2}$ & $-\omega^{2}$ \\
\hline$\widetilde{4}^{\prime \prime}$ & 0 & $\omega^{2}$ & $\omega^{2}$ & $\omega^{2}$ & $\omega^{2}$ & $-\omega^{2}$ & $-\omega^{2}$ & $\omega^{2}$ & $-\omega^{2}$ & $\omega^{2}$ & $\omega$ & $\omega$ & $\omega$ & $\omega$ & $-\omega$ & $-\omega$ & $\omega$ & $-\omega$ \\
\hline 6 & -2 & 0 & 0 & 0 & 0 & 0 & 0 & 0 & 0 & 0 & 0 & 0 & 0 & 0 & 0 & 0 & 0 & 0 \\
\hline $6^{\prime}$ & 2 & 0 & 0 & 0 & 0 & 0 & 0 & 0 & 0 & 0 & 0 & 0 & 0 & 0 & 0 & 0 & 0 & 0 \\
\hline 8 & 0 & 2 & -1 & -1 & 0 & 0 & -1 & 2 & 2 & 0 & 2 & -1 & -1 & 0 & 0 & -1 & 2 & 2 \\
\hline $8^{\prime}$ & 0 & $2 \omega$ & $-\omega$ & $-\omega$ & 0 & 0 & $-\omega$ & $2 \omega$ & $2 \omega$ & 0 & $2 \omega^{2}$ & $-\omega^{2}$ & $-\omega^{2}$ & 0 & 0 & $-\omega^{2}$ & $2 \omega^{2}$ & $2 \omega^{2}$ \\
\hline $8^{\prime \prime}$ & 0 & $2 \omega^{2}$ & $-\omega^{2}$ & $-\omega^{2}$ & 0 & 0 & $-\omega^{2}$ & $2 \omega^{2}$ & $2 \omega^{2}$ & 0 & $2 \omega$ & $-\omega$ & $-\omega$ & 0 & 0 & $-\omega$ & $2 \omega$ & $2 \omega$ \\
\hline$\widetilde{8}$ & 0 & 2 & -1 & -1 & 0 & 0 & 1 & 2 & -2 & 0 & 2 & -1 & -1 & 0 & 0 & 1 & 2 & -2 \\
\hline$\widetilde{8}^{\prime}$ & 0 & $2 \omega$ & $-\omega$ & $-\omega$ & 0 & 0 & $\omega$ & $2 \omega$ & $-2 \omega$ & 0 & $2 \omega^{2}$ & $-\omega^{2}$ & $-\omega^{2}$ & 0 & 0 & $\omega^{2}$ & $2 \omega^{2}$ & $-2 \omega^{2}$ \\
\hline$\widetilde{8}^{\prime \prime}$ & 0 & $2 \omega^{2}$ & $-\omega^{2}$ & $-\omega^{2}$ & 0 & 0 & $\omega^{2}$ & $2 \omega^{2}$ & $-2 \omega^{2}$ & 0 & $2 \omega$ & $-\omega$ & $-\omega$ & 0 & 0 & $\omega$ & $2 \omega$ & $-2 \omega$ \\
\hline 16 & 0 & 4 & -2 & 1 & 0 & 0 & 0 & -2 & 0 & 0 & 4 & -2 & 1 & 0 & 0 & 0 & -2 & 0 \\
\hline $16^{\prime}$ & 0 & $4 \omega$ & $-2 \omega$ & $\omega$ & 0 & 0 & 0 & $-2 \omega$ & 0 & 0 & $4 \omega^{2}$ & $-2 \omega^{2}$ & $\omega^{2}$ & 0 & 0 & 0 & $-2 \omega^{2}$ & 0 \\
\hline $16^{\prime \prime}$ & 0 & $4 \omega^{2}$ & $-2 \omega^{2}$ & $\omega^{2}$ & 0 & 0 & 0 & $-2 \omega^{2}$ & 0 & 0 & $4 \omega$ & $-2 \omega$ & $\omega$ & 0 & 0 & 0 & $-2 \omega$ & 0 \\
\hline 24 & 0 & 0 & 0 & 0 & 0 & 0 & 0 & 0 & 0 & 0 & 0 & 0 & 0 & 0 & 0 & 0 & 0 & 0 \\
\hline $24^{\prime}$ & 0 & 0 & 0 & 0 & 0 & 0 & 0 & 0 & 0 & 0 & 0 & 0 & 0 & 0 & 0 & 0 & 0 & 0 \\
\hline 24 & 0 & 0 & 0 & 0 & 0 & 0 & 0 & 0 & 0 & 0 & 0 & 0 & 0 & 0 & 0 & 0 & 0 & 0 \\
\hline$\widetilde{24}^{\prime}$ & 0 & 0 & 0 & 0 & 0 & 0 & 0 & 0 & 0 & 0 & 0 & 0 & 0 & 0 & 0 & 0 & 0 & 0 \\
\hline $24^{\prime \prime}$ & 0 & 0 & 0 & 0 & 0 & 0 & 0 & 0 & 0 & 0 & 0 & 0 & 0 & 0 & 0 & 0 & 0 & 0 \\
\hline $24^{\prime \prime \prime}$ & 0 & 0 & 0 & 0 & 0 & 0 & 0 & 0 & 0 & 0 & 0 & 0 & 0 & 0 & 0 & 0 & 0 & 0 \\
\hline 32 & 0 & 2 & 2 & -1 & 2 & 0 & 0 & -1 & 0 & -1 & 2 & 2 & -1 & 2 & 0 & 0 & -1 & 0 \\
\hline $32^{\prime}$ & 0 & $2 \omega$ & $2 \omega$ & $-\omega$ & $2 \omega$ & 0 & 0 & $-\omega$ & 0 & $-\omega$ & $2 \omega^{2}$ & $2 \omega^{2}$ & $-\omega^{2}$ & $2 \omega^{2}$ & 0 & 0 & $-\omega^{2}$ & 0 \\
\hline $32^{\prime \prime}$ & 0 & $2 \omega^{2}$ & $2 \omega^{2}$ & $-\omega^{2}$ & $2 \omega^{2}$ & 0 & 0 & $-\omega^{2}$ & 0 & $-\omega^{2}$ & $2 \omega$ & $2 \omega$ & $-\omega$ & $2 \omega$ & 0 & 0 & $-\omega$ & 0 \\
\hline$\widetilde{32}$ & 0 & 2 & 2 & -1 & -2 & 0 & 0 & -1 & 0 & 1 & 2 & 2 & -1 & -2 & 0 & 0 & -1 & 0 \\
\hline$\widetilde{32}^{\prime}$ & 0 & $2 \omega$ & $2 \omega$ & $-\omega$ & $-2 \omega$ & 0 & 0 & $-\omega$ & 0 & $\omega$ & $2 \omega^{2}$ & $2 \omega^{2}$ & $-\omega^{2}$ & $-2 \omega^{2}$ & 0 & 0 & $-\omega^{2}$ & 0 \\
\hline$\widetilde{32}^{\prime \prime}$ & 0 & $2 \omega^{2}$ & $2 \omega^{2}$ & $-\omega^{2}$ & $-2 \omega^{2}$ & 0 & 0 & $-\omega^{2}$ & 0 & $\omega^{2}$ & $2 \omega$ & $2 \omega$ & $-\omega$ & $-2 \omega$ & 0 & 0 & $-\omega$ & 0 \\
\hline 48 & 0 & 0 & 0 & 0 & 0 & 0 & 0 & 0 & 0 & 0 & 0 & 0 & 0 & 0 & 0 & 0 & 0 & 0 \\
\hline$\widetilde{48}$ & 0 & 0 & 0 & 0 & 0 & 0 & 0 & 0 & 0 & 0 & 0 & 0 & 0 & 0 & 0 & 0 & 0 & 0 \\
\hline
\end{tabular}

Table 2: The character table for $\left(S_{3} \times S_{3} \times S_{3} \times S_{3}\right) \rtimes A_{4}$. 


\subsection{Lepton assignment}

\begin{tabular}{|c|c|c|c|c|}
\hline & $S U(2)_{L}$ & $U(1)_{Y}$ & $S_{3}^{4} \rtimes A_{4}$ & $Z_{2}$ \\
\hline$L$ & 2 & $-1 / 2$ & 3 & +1 \\
$e_{R 1}$ & 1 & -1 & 1 & -1 \\
$e_{R 2}$ & 1 & -1 & $1^{\prime}$ & -1 \\
$e_{R 3}$ & 1 & -1 & $1^{\prime \prime}$ & -1 \\
$N$ & 1 & 0 & 3 & +1 \\
$N^{\prime}$ & 1 & 0 & 48 & +1 \\
$N^{\prime \prime}$ & 1 & 0 & 8 & +1 \\
$E_{L}$ & 1 & -1 & 3 & +1 \\
$E_{R}$ & 1 & -1 & 3 & +1 \\
$\phi$ & 1 & 0 & 16 & +1 \\
$\chi$ & 1 & 0 & 3 & -1 \\
$H$ & 2 & $-1 / 2$ & 1 & +1 \\
\hline
\end{tabular}

Table 3: This table shows the assignments of the fermions and Higgs fields under $S U(2)_{L} \times$ $U(1)_{Y} \times\left(S_{3}^{4} \rtimes A_{4}\right) \times Z_{2}$.

The leptonic and scalar particle content of the model in is given in Table 3. In more detail, the SM leptons transform under $\left(S_{3}^{4} \rtimes A_{4}\right) \times Z_{2}$ as

$$
e_{R 1} \sim(1,-1), \quad e_{R 2} \sim\left(1^{\prime},-1\right), \quad e_{R 3} \sim\left(1^{\prime \prime},-1\right), \quad\left(L_{1}, L_{2}, L_{3}\right) \sim(3,+1) .
$$

Here the second entry indicates $Z_{2}$ transformation of the field. The $A_{4}$ assignment is identical to the one in Ref. [5, 6, 7, 8, 9, 10, 11, 12, 13, 14. The charged lepton masses are generated by effective interactions involving a real Higgs multiplet $\chi$ belonging to a $Z_{2}$ odd triplet of $A_{4}$. This involves integrating out multiplets of heavy vector-like fermions, whose masses are at the high scale $M_{*}$, with the same gauge quantum numbers as right-handed charged leptons. These are $E_{L, R} \sim(3,+1)$ under $\left(S_{3}^{4} \rtimes A_{4}\right) \times Z_{2}$. The Yukawa interactions for the charged leptons are given by

$$
\begin{aligned}
\mathcal{L}_{e} & =\kappa f_{2}\left(\bar{E}_{R}, L\right) H+m_{E} f_{2}\left(\bar{E}_{R}, E_{L}\right)+\epsilon_{1} \bar{e}_{R 1} f_{2}\left(E_{L}, \chi\right) \\
& +\epsilon_{2} g_{5}\left(\bar{e}_{R 2}, E_{L}, \chi\right)+\epsilon_{3} g_{6}\left(\bar{e}_{R 3}, E_{L}, \chi\right)+\text { c.c. },
\end{aligned}
$$

where the functions $f_{2}, g_{5}$, and $g_{6}$ are given in Appendix B. Here $H$ is the SM Higgs doublet.

Neutrino Dirac masses are generated by effective interactions involving a real Higgs multiplet $\phi$ belonging to a $Z_{2}$ even 16-dimensional representation of $S_{3}^{4} \rtimes A_{4}$. This involves integrating out multiplets of heavy right-handed neutrinos, with masses at the high scale $M_{*}$. These multiplets are $N \sim(3,+1), N^{\prime} \sim(48,+1)$, and $N^{\prime \prime} \sim(8,+1)$ under $\left(S_{3}^{4} \rtimes A_{4}\right) \times Z_{2}$. The 
Yukawa interactions for the neutrinos are given by

$$
\begin{aligned}
\mathcal{L}_{\nu} & =\lambda f_{2}(L, N) \widetilde{H}+m_{N} f_{2}(N, N)+m_{N}^{\prime} f_{3}\left(N^{\prime}, N^{\prime}\right)+m_{N}^{\prime \prime} f_{4}\left(N^{\prime \prime}, N^{\prime \prime}\right) \\
& +\alpha_{1} g_{2}\left(N, \phi, N^{\prime}\right)+\alpha_{2} g_{3}\left(N^{\prime \prime}, \phi, N^{\prime}\right)+\beta g_{4}\left(\phi, N^{\prime}, N^{\prime}\right)
\end{aligned}
$$

where the functions $f_{2}, f_{3}, f_{4}, g_{2}, g_{3}$, and $g_{4}$ are given in Appendix B, and where $\widetilde{H}=i \tau_{2} H^{*}$. Interactions involving $\chi$, such as $N N \chi$ and $N^{\prime} N^{\prime} \chi$, are allowed by $S_{3}^{4} \rtimes A_{4}$, but are prevented by the $Z_{2}$ symmetry.

As will be shown in the subsequent sections, these leptonic interactions will lead to the desired tribimaximal pattern of mixing, without any vacuum alignment problem. Symmetryinvariant interactions between $\phi$ and $\chi$ must consist of products of $S_{3}^{4}$ invariants constructed from $\phi$ with $Z_{2}$ invariants constructed from $\chi$. The 16-dimensional representation to which $\phi$ belongs is $(2,2,2,2)$ with respect to $S_{3}^{4}$. (Since $(2,2,2,2)$ is invariant under permutations, it does not mix with other representations when $S_{3}^{4}$ is embedded into $S_{3}^{4} \rtimes A_{4}$.) There is only one quadratic $S_{3}^{4}$ invariant that can be constructed with $\phi$, which is a 1 of $A_{4}$. So there is no $16 \times 16 \times 3$ invariant. There is also only one cubic $S_{3}^{4}$ invariant that can be constructed with $\phi$, which is also a 1 of $A_{4}$. So there is no $16 \times 16 \times 16 \times 3$ invariant. Thus, there are no cubic invariants involving both $\phi$ and $\chi$, and the only quartic invariant containing both is a product of quadratic invariants, which does not generate a VEV alignment problem. However, $16 \times 16 \times 16 \times 16$ does contain not only 3 but also $1^{\prime}$ and $1^{\prime \prime}$, so that non-trivial $\phi^{4} \chi^{2}$ invariants do exist, but these are non-renormalizable interactions, and are presumably suppressed by the Planck scale. Inclusion of such suppressed operators will have no significant effects on the the desired vacuum structure.

It is vitally important here that the 16 contains only one complete multiplet under the first factor, $S_{3}^{4}$, of the semi-direct product. If an abelian group were used for the first factor, then a representation that does not mix like this would have to be one-dimensional. But it would be impossible to obtain the symmetry breaking pattern we desire with a one-dimensional representation. (A one-dimensional representation can only break a group to an invariant subgroup, but the $Z_{2}$ and $Z_{3}$ subgroups of $A_{4}$, referred to in the introduction, that we desire are not invariant.) So, a non-abelian group is required, and $S_{3}$ is the simplest non-abelian group. Four factors are used so that a non-trivial semi-direct product with $A_{4}$ can be taken.

\section{Higgs potential and its minimum}

The potential involving the $\phi$ and $\chi$ fields has the form

$$
\begin{aligned}
V_{\phi \chi} & =a_{1} f_{1}(\phi, \phi)+a_{2} f_{2}(\chi, \chi)+b g_{1}(\phi, \phi, \phi)+c_{1} h_{1}(\phi)+c_{2} h_{2}(\phi) \\
& +c_{3} h_{3}(\chi)+c_{4} h_{4}(\chi)+c_{5} f_{1}(\phi, \phi) f_{2}(\chi, \chi)
\end{aligned}
$$

where the functions $f_{1}, f_{2}, g_{1}, h_{1}, h_{2}, h_{3}$, and $h_{4}$ are given in Appendix B. 
If the Higgs potential in (111) is to be bounded below, the following constraints on the parameters must be satisfied:

$$
\begin{aligned}
& c_{1}>0, \quad c_{1}+2 c_{2}>0, \quad c_{3}>0, \quad c_{3}+c_{4}>0, \\
& c_{5}+\sqrt{c_{1} c_{3}}>0, \quad c_{5}+\frac{1}{2} \sqrt{\frac{1}{6}\left(c_{1}+2 c_{2}\right)\left(c_{3}+c_{4}\right)}>0 .
\end{aligned}
$$

Upon minimizing the potential,

$$
\begin{aligned}
& \langle\chi\rangle=\left(v_{\chi}, v_{\chi}, v_{\chi}\right) \\
& \langle\phi\rangle=\left(0,0,0,0, v_{\phi}, v_{\phi}, v_{\phi}, v_{\phi}, v_{\phi}, v_{\phi}, v_{\phi}, v_{\phi}, 0,0,0,0\right)
\end{aligned}
$$

is found to be an extremum for

$$
2 a_{1}+3 b_{1} v_{\phi}+2\left(c_{1}+c_{2}\right) v_{\phi}^{2}+6 c_{5} v_{\chi}^{2}=0, \quad a_{2}+4\left(c_{3}+c_{4}\right) v_{\chi}^{2}+8 c_{5} v_{\phi}^{2}=0 .
$$

The Higgs boson masses are found to be

$$
\begin{gathered}
3 b v_{\phi}+4\left(c_{1}+c_{2}\right) v_{\phi}^{2}+8\left(c_{3}+c_{4}\right) v_{\chi}^{2} \pm \sqrt{\left[3 b v_{\phi}+4\left(c_{1}+c_{2}\right) v_{\phi}^{2}-8\left(c_{3}+c_{4}\right) v_{\chi}^{2}\right]^{2}+1536 c_{5}^{2} v_{\phi}^{2} v_{\chi}^{2}} \\
-18 b v_{\phi}, \quad-6 b v_{\phi}-4 c_{1} v_{\phi}^{2}, \quad 6 b v_{\phi}+8 c_{1} v_{\phi}^{2}, \quad 6 b v_{\phi}+8\left(c_{1}-c_{2}\right) v_{\phi}^{2}, \quad 8\left(2 c_{3}-c_{4}\right) v_{\chi}^{2} .
\end{gathered}
$$

Each mass in the first line occurs once, while those in the second line occur four, eight, two, one, and two times respectively. Assuming (12), we see that (13) is a local minimum for

$$
\begin{aligned}
& b v_{\phi}<0, \quad \frac{2}{3}<\left|\frac{b}{c_{1} v_{\phi}}\right|<\frac{4}{3}, \quad-1<\frac{c_{3}}{c_{4}}<2, \quad-\frac{1}{3}<\frac{c_{2} / c_{1}}{4-3\left|\frac{b}{c_{1} v_{\phi}}\right|}<1, \\
& \left|c_{5}\right|<\frac{1}{4} \sqrt{\frac{1}{3}\left[\frac{3 b}{v_{\phi}}+4\left(c_{1}+c_{2}\right)\right]\left(c_{3}+c_{4}\right)} .
\end{aligned}
$$

The VEV of $\chi$ in (13) breaks $A_{4}$ to the $Z_{3}$ subgroup of $A_{4}$ generated by $Y$. Note also that $S_{3}^{4}$ is left unbroken by $\chi$ trivially, but $Z_{2}$ is broken. On the other hand, the VEV of $\phi$ breaks $A_{4}$ to the $Z_{2}$ subgroup of $A_{4}$ generated by $X$. Additionally, $\phi$ leaves the generators $B_{1}, B_{2}$, $B_{3} B_{4}$, and $A_{3} A_{4}$ unbroken. These form the subgroup $D_{4} \times S_{3}$, with $D_{4}$ generated by $B_{1}, B_{2}$, and $X$ and with $S_{3}$ generated by $A_{3} A_{4}$ and $B_{3} B_{4}$. This VEV also trivially leaves the additional external $Z_{2}$ unbroken.

Note that the Higgs potential does not have any accidental global symmetries, which would have resulted in pseudo-Goldstone bosons. We conclude that consistent symmetry breaking can be realized without any vacuum alignment problem. 


\section{Lepton masses}

The light lepton masses can now be readily inferred from the Lagrangian of (91) and (10), along with the VEV structure of $\chi$ and $\phi$ given in (13). The Higgs field $\chi$ mixes $E_{L, R}$ with the SM charged leptons in (9), generating the light charged lepton mass matrix. Upon integrating out $E_{L, R}$, the $Z_{3}$ subgroup of $A_{4}$ left unbroken by the VEV of $\chi$ forces the light left-handed charged lepton mass matrix to have the form (see Appendix C for details)

$$
\begin{gathered}
M_{e}^{\dagger} M_{e}=\frac{1}{\sqrt{3}}\left(\begin{array}{ccc}
1 & 1 & 1 \\
1 & \omega & \omega^{2} \\
1 & \omega^{2} & \omega
\end{array}\right)\left(\begin{array}{ccc}
m_{e}^{2} & 0 & 0 \\
0 & m_{\mu}^{2} & 0 \\
0 & 0 & m_{\tau}^{2}
\end{array}\right) \frac{1}{\sqrt{3}}\left(\begin{array}{ccc}
1 & 1 & 1 \\
1 & \omega^{2} & \omega \\
1 & \omega & \omega^{2}
\end{array}\right) \\
=U_{L}\left(\begin{array}{ccc}
m_{e}^{2} & 0 & 0 \\
0 & m_{\mu}^{2} & 0 \\
0 & 0 & m_{\tau}^{2}
\end{array}\right) U_{L}^{\dagger} .
\end{gathered}
$$

The masses are given by

$$
m_{i}^{2}=\frac{3\left|\kappa \epsilon_{i} v_{\chi} v\right|^{2}}{3\left|\epsilon_{i} v_{\chi}\right|^{2}+\left|m_{E}\right|^{2}} .
$$

The Higgs field $\phi$ mixes the heavy right-handed neutrinos with the light left-handed neutrinos in (10), generating the light neutrino mass matrix. $N \sim 3$ is required because the SM Higgs $H$ only breaks EW symmetry, so that it can only cause left-handed neutrinos to mix with a triplet. Since $3 \times 16=48, \phi \sim 16$ induces mixing between $N$ and $N^{\prime} \sim 48 . N^{\prime \prime} \sim 8$ is needed to remove unwanted accidental symmetries. Upon integrating out the heavy right-handed neutrinos, the $Z_{2}$ subgroup of $A_{4}$ left unbroken by the VEV of $\phi$ (along with an additional accidental $Z_{2}$ that is actually part of $S_{4}$ [17]) forces the light neutrino mass matrix to have the form (see Appendix D)

$$
M_{\nu}=\left(\begin{array}{ccc}
a_{\nu} & 0 & c_{\nu} \\
0 & b_{\nu} & 0 \\
c_{\nu} & 0 & a_{\nu}
\end{array}\right) .
$$

This matrix is diagonalized by

$$
U_{\nu}=\frac{1}{\sqrt{2}}\left(\begin{array}{ccc}
1 & 0 & -1 \\
0 & \sqrt{2} & 0 \\
1 & 0 & 1
\end{array}\right) P_{\nu}
$$


where diagonal $P_{\nu}$ is a phase matrix. The light neutrino masses are found to be

$$
\begin{aligned}
& m_{1}=\left|\frac{\lambda^{2} v^{2}}{2} \frac{m_{N}^{\prime} m_{N}^{\prime \prime}-4 \alpha_{2}^{2} v_{\phi}^{2}+\beta v_{\phi} m_{N}^{\prime \prime}}{-2 \alpha_{1}^{2} v_{\phi}^{2}+m_{N}\left(m_{N}^{\prime} m_{N}^{\prime \prime}-4 \alpha_{2}^{2} v_{\phi}^{2}+\beta v_{\phi} m_{N}^{\prime \prime}\right)}\right|, \\
& m_{2}=\left|\frac{\lambda^{2} v^{2}}{2} \frac{m_{N}^{\prime} m_{N}^{\prime \prime}-2 \alpha_{2}^{2} v_{\phi}^{2}+\beta v_{\phi} m_{N}^{\prime \prime}}{-2 \alpha_{1}^{2} v_{\phi}^{2}+m_{N}\left(m_{N}^{\prime} m_{N}^{\prime \prime}-2 \alpha_{2}^{2} v_{\phi}^{2}+\beta v_{\phi} m_{N}^{\prime \prime}\right)}\right|, \\
& m_{3}=\left|\frac{\lambda^{2} v^{2}}{2} \frac{m_{N}^{\prime}+\beta v_{\phi}}{-2 \alpha_{1}^{2} v_{\phi}^{2}+m_{N}\left(m_{N}^{\prime}+\beta v_{\phi}\right)}\right| .
\end{aligned}
$$

Note that the three neutrino masses given in (21) are unrelated. This spectrum allows for normal neutrino mass hierarchy, inverted mass hierarchy, as well as quasi-degenerate neutrinos. The last possibility is realized when the bare mass parameters in (21) are taken to be slightly larger than the $\mathrm{VEV} v_{\phi}$.

Eqs. (17) and (20) give the desired form (2) for the mixing matrix $U_{M N S}=U_{L}^{T} U_{\nu}^{*}$.

\section{Inclusion of quarks}

We have not yet specified how quarks transform under the finite family symmetry. One possibility is to have the quarks not transform under this symmetry at all, in which case the quark sector is exactly like the SM. Another, perhaps more interesting, possibility is to use an assignment analogous to that of the charged leptons,

$$
u_{R 1}, d_{R 1} \sim(1,-1), \quad u_{R 2}, d_{R 2} \sim\left(1^{\prime},-1\right), \quad u_{R 3}, d_{R 3} \sim\left(1^{\prime \prime},-1\right), \quad\left(Q_{1}, Q_{2}, Q_{3}\right)_{L} \sim(3,+1) .
$$

Then, add additional heavy quark multiplets $U_{L, R}$ and $D_{L, R}$, transforming as $(3,1,+2 / 3,3,+1)$ and $(3,1,-1 / 3,3,+1)$ respectively under $S U(3)_{c} \times S U(2)_{L} \times U(1)_{Y} \times\left(S_{3}^{4} \rtimes A_{4}\right) \times Z_{2}$. For the up-type quarks, we obtain the Yukawa Lagrangian

$$
\begin{aligned}
\mathcal{L}_{u} & =\kappa^{\prime} f_{2}\left(\bar{U}_{R}, Q_{L}\right) H+m_{U} f_{2}\left(\bar{U}_{R}, U_{L}\right)+\epsilon_{1}^{\prime} \bar{u}_{R 1} f_{2}\left(U_{L}, \chi\right) \\
& +\epsilon_{2}^{\prime} g_{5}\left(\bar{u}_{R 2}, U_{L}, \chi\right)+\epsilon_{3}^{\prime} g_{6}\left(\bar{u}_{R 3}, U_{L}, \chi\right)+\text { c.c. },
\end{aligned}
$$

with a similar result for down-type quarks. This gives non-degenerate quark masses but no mixing.

Small and arbitrary quark mixing can be generated from $\phi$ by adding additional heavy quark multiplets:

$$
\begin{aligned}
& U_{1 L, R}^{\prime} \sim(3,1,+2 / 3,16,-1), \quad U_{2 L, R}^{\prime} \sim\left(3,1,+2 / 3,16^{\prime},-1\right), \\
& U_{3 L, R}^{\prime} \sim\left(3,1,+2 / 3,16^{\prime \prime},-1\right), \quad U_{L, R}^{\prime \prime} \sim(3,1,+2 / 3,6,-1) .
\end{aligned}
$$

The generators for these representations can be found in Appendix A. Heavy quarks can be added to the down-type sector instead, but to generate mixing it is only necessary to do this 
for one sector. The up-type Yukawa Lagrangian receives the additional contribution

$$
\begin{aligned}
\mathcal{L}_{u}^{\prime} & =m_{1 U}^{\prime} f_{1}\left(\bar{U}_{1 R}^{\prime}, U_{1 L}^{\prime}\right)+m_{2 U}^{\prime} f_{1}\left(\bar{U}_{2 R}^{\prime}, U_{2 L}^{\prime}\right)+m_{3 U}^{\prime} f_{1}\left(\bar{U}_{3 R}^{\prime}, U_{3 L}^{\prime}\right)+m_{U}^{\prime \prime} f_{5}\left(\bar{U}_{R}^{\prime \prime}, U_{L}^{\prime \prime}\right) \\
& +\delta_{1} \bar{u}_{R 1} f_{2}\left(U_{L}^{\prime}, \phi\right)+\delta_{2} \bar{u}_{R 2} f_{2}\left(U_{L}^{\prime}, \phi\right)+\delta_{3} \bar{u}_{R 3} f_{2}\left(U_{L}^{\prime}, \phi\right)+\zeta_{1} g_{7}\left(\bar{U}_{R}^{\prime \prime}, U_{1 L}^{\prime}, \phi\right)+\zeta_{2} g_{8}\left(\bar{U}_{R}^{\prime \prime}, U_{2 L}^{\prime}, \phi\right) \\
& +\zeta_{3} g_{9}\left(\bar{U}_{R}^{\prime \prime}, U_{3 L}^{\prime}, \phi\right)+\xi_{1} g_{7}\left(\bar{U}_{L}^{\prime \prime}, U_{1 R}^{\prime}, \phi\right)+\xi_{2} g_{8}\left(\bar{U}_{L}^{\prime \prime}, U_{2 R}^{\prime}, \phi\right)+\xi_{3} g_{9}\left(\bar{U}_{L}^{\prime \prime}, U_{3 R}^{\prime}, \phi\right),
\end{aligned}
$$

where the functions $f_{5}, g_{7}, g_{8}$, and $g_{9}$ can be found in Appendix B. In this way, arbitrary quark mixing can be obtained.

\section{Supersymmetrization}

Supersymmetrization of the $\left(S_{3}\right)^{4} \rtimes A_{4}$ sector of the model is straightforward. Since in a renormaliable SUSY model one uses only quadratic and cubic (and not quartic) invariants in the superpotential, care should be given to ensure that there are no flat directions. The $Z_{2}$ factor in the model of Sec. II would prevent a $\chi^{3}$ invariant. This will leads to flat directions in the cubic superpotential of the SUSY version of the model. If one wishes to avoid increasing the number of Higgs multiplets, the problem can be solved by replacing $Z_{2}$ with some other symmetry. So, consider $\left(S_{3}^{4} \rtimes A_{4}\right) \times H$. It's not difficult to see that for $H=Z_{n}$, the flat direction problem is not solved. The next simplest possibility is to consider $\chi \sim(3,2)$ of $\left(S_{3}^{4} \rtimes A_{4}\right) \times H$, with $H$ a $D_{n}$ or $Q_{n}$. However, one finds that the flat direction problem persists for representations such as these. So, we are led to consider $\left(S_{3}^{4} \rtimes A_{4}\right) \times A_{4}$ with $\chi \sim(3,3)$. It is easily confirmed that the $(3,3)$ cubic superpotential has no flat directions. The light charged lepton masses can be generated from this $\chi$ by integrating out two sets of heavy vector-like superfields with the same gauge quantum numbers as right-handed charged leptons, transforming as $(3,1)$ and $(3,3)$ under $\left(S_{3}^{4} \rtimes A_{4}\right) \times A_{4}$. Alternatively, one can solve the flat direction problem by increasing the number on Higgs multiplets.

The SUSY flavor problem can be readily solved by the symmetry $G=\left(S_{3}^{4} \rtimes A_{4}\right) \times A_{4}$. The SM lepton and quark doublets belong to triplet representations of the $A_{4}$ factor group, which would mean that the soft SUSY breaking masses of all three families of sleptons (and similarly squarks) are the same. As for the right-handed sleptons and squarks, the group $G$ does allow the three families to have non-degenerate soft masses. However, there is no mixing in the right-handed slepton mass matrix, which is sufficient to ensure the absence of excessive SUSY flavor violation. Since there is mixing between the right-handed singlet leptons and heavy vector-like leptons of order $v_{\chi, \phi} / M_{*}$, adequate suppression of SUSY FCNC would require that $v_{\chi, \phi}$ be somewhat smaller than $M_{*}$.

\section{Summary and conclusions}

First, we presented a renormalizable non-supersymmetric model based on the finite symmetry $\left(S_{3}^{4} \rtimes A_{4}\right) \times Z_{2}$, with the SM leptons assigned to representations of $A_{4}$. Neutrino masses are 
generated by a Higgs field $\phi$ belonging to a $Z_{2}$ even 16-dimensional representation of $S_{3}^{4} \rtimes A_{4}$ while charged-lepton masses are generated by a Higgs field $\chi$ belonging to a $Z_{2}$ odd triplet representation of $A_{4}$. The additional symmetries, $S_{3}^{4}$ and $Z_{2}$, prevent quadratic and cubic interactions between $\phi$ and $\chi$ and allow only a trivial quartic interaction that does not cause an alignment problem, addressing the alignment problem without altering the desired properties of the family symmetry. In this way, we are able to explain all aspects of neutrino mixing using only symmetries which are spontaneously broken by the Higgs mechanism.

\section{Appendix A}

In this appendix, we give the explicit matrices representing the generators in Eqs. (44)-(7) for each representation used in our model. These matrices will be used for computing the group invariants, given in Appendix B. The generators for 16, in a certain basis, are

$$
\begin{aligned}
A_{1}^{(16)}=\operatorname{diag}\left(\omega, \omega^{2}, \omega, \omega^{2}, \omega, \omega^{2}, \omega, \omega^{2}, \omega, \omega^{2}, \omega, \omega^{2}, \omega, \omega^{2}, \omega, \omega^{2}\right), \\
A_{3}^{(16)}=\operatorname{diag}\left(\omega, \omega, \omega^{2}, \omega^{2}, \omega, \omega, \omega^{2}, \omega^{2}, \omega, \omega, \omega^{2}, \omega^{2}, \omega, \omega, \omega^{2}, \omega^{2}\right), \\
A_{4}^{(16)}=\operatorname{diag}\left(\omega, \omega, \omega, \omega, \omega^{2}, \omega^{2}, \omega^{2}, \omega^{2}, \omega, \omega, \omega, \omega, \omega^{2}, \omega^{2}, \omega^{2}, \omega^{2}\right), \\
B_{1}^{(16)}= \\
\left.\left.\begin{array}{llllllllllllllllll}
16 & \\
0 & 1 & 0 & 0 & 0 & 0 & 0 & 0 & 0 & 0 & 0 & 0 & 0 & 0 & 0 & 0 & 0 \\
0 & 0 & 0 & 1 & 0 & 0 & 0 & 0 & 0 & 0 & 0 & 0 & 0 & 0 & 0 & 0 \\
0 & 0 & 1 & 0 & 0 & 0 & 0 & 0 & 0 & 0 & 0 & 0 & 0 & 0 & 0 & 0 \\
0 & 0 & 0 & 0 & 0 & 1 & 0 & 0 & 0 & 0 & 0 & 0 & 0 & 0 & 0 & 0 \\
0 & 0 & 0 & 0 & 1 & 0 & 0 & 0 & 0 & 0 & 0 & 0 & 0 & 0 & 0 & 0 \\
0 & 0 & 0 & 0 & 0 & 0 & 0 & 1 & 0 & 0 & 0 & 0 & 0 & 0 & 0 & 0 \\
0 & 0 & 0 & 0 & 0 & 0 & 1 & 0 & 0 & 0 & 0 & 0 & 0 & 0 & 0 & 0 \\
0 & 0 & 0 & 0 & 0 & 0 & 0 & 0 & 0 & 1 & 0 & 0 & 0 & 0 & 0 & 0 \\
0 & 0 & 0 & 0 & 0 & 0 & 0 & 0 & 1 & 0 & 0 & 0 & 0 & 0 & 0 & 0 \\
0 & 0 & 0 & 0 & 0 & 0 & 0 & 0 & 0 & 0 & 0 & 1 & 0 & 0 & 0 & 0 \\
0 & 0 & 0 & 0 & 0 & 0 & 0 & 0 & 0 & 0 & 1 & 0 & 0 & 0 & 0 & 0 \\
0 & 0 & 0 & 0 & 0 & 0 & 0 & 0 & 0 & 0 & 0 & 0 & 0 & 1 & 0 & 0 \\
0 & 0 & 0 & 0 & 0 & 0 & 0 & 0 & 0 & 0 & 0 & 0 & 1 & 0 & 0 & 0 \\
0 & 0 & 0 & 0 & 0 & 0 & 0 & 0 & 0 & 0 & 0 & 0 & 0 & 0 & 0 & 1 \\
0 & 0 & 0 & 0 & 0 & 0 & 0 & 0 & 0 & 0 & 0 & 0 & 0 & 0 & 1 & 0
\end{array}\right), \omega, \omega, \omega^{2}, \omega^{2}, \omega^{2}, \omega^{2}, \omega^{2}, \omega^{2}, \omega^{2}, \omega^{2}\right),
\end{aligned}
$$




$$
B_{2}^{(16)}=\left(\begin{array}{cccccccccccccccc}
0 & 0 & 1 & 0 & 0 & 0 & 0 & 0 & 0 & 0 & 0 & 0 & 0 & 0 & 0 & 0 \\
0 & 0 & 0 & 1 & 0 & 0 & 0 & 0 & 0 & 0 & 0 & 0 & 0 & 0 & 0 & 0 \\
1 & 0 & 0 & 0 & 0 & 0 & 0 & 0 & 0 & 0 & 0 & 0 & 0 & 0 & 0 & 0 \\
0 & 1 & 0 & 0 & 0 & 0 & 0 & 0 & 0 & 0 & 0 & 0 & 0 & 0 & 0 & 0 \\
0 & 0 & 0 & 0 & 0 & 0 & 1 & 0 & 0 & 0 & 0 & 0 & 0 & 0 & 0 & 0 \\
0 & 0 & 0 & 0 & 0 & 0 & 0 & 1 & 0 & 0 & 0 & 0 & 0 & 0 & 0 & 0 \\
0 & 0 & 0 & 0 & 1 & 0 & 0 & 0 & 0 & 0 & 0 & 0 & 0 & 0 & 0 & 0 \\
0 & 0 & 0 & 0 & 0 & 1 & 0 & 0 & 0 & 0 & 0 & 0 & 0 & 0 & 0 & 0 \\
0 & 0 & 0 & 0 & 0 & 0 & 0 & 0 & 0 & 0 & 1 & 0 & 0 & 0 & 0 & 0 \\
0 & 0 & 0 & 0 & 0 & 0 & 0 & 0 & 0 & 0 & 0 & 1 & 0 & 0 & 0 & 0 \\
0 & 0 & 0 & 0 & 0 & 0 & 0 & 0 & 1 & 0 & 0 & 0 & 0 & 0 & 0 & 0 \\
0 & 0 & 0 & 0 & 0 & 0 & 0 & 0 & 0 & 1 & 0 & 0 & 0 & 0 & 0 & 0 \\
0 & 0 & 0 & 0 & 0 & 0 & 0 & 0 & 0 & 0 & 0 & 0 & 0 & 0 & 1 & 0 \\
0 & 0 & 0 & 0 & 0 & 0 & 0 & 0 & 0 & 0 & 0 & 0 & 0 & 0 & 0 & 1 \\
0 & 0 & 0 & 0 & 0 & 0 & 0 & 0 & 0 & 0 & 0 & 0 & 1 & 0 & 0 & 0 \\
0 & 0 & 0 & 0 & 0 & 0 & 0 & 0 & 0 & 0 & 0 & 0 & 0 & 1 & 0 & 0
\end{array}\right),
$$

$$
B_{3}^{(16)}=\left(\begin{array}{cccccccccccccccc}
0 & 0 & 0 & 0 & 1 & 0 & 0 & 0 & 0 & 0 & 0 & 0 & 0 & 0 & 0 & 0 \\
0 & 0 & 0 & 0 & 0 & 1 & 0 & 0 & 0 & 0 & 0 & 0 & 0 & 0 & 0 & 0 \\
0 & 0 & 0 & 0 & 0 & 0 & 1 & 0 & 0 & 0 & 0 & 0 & 0 & 0 & 0 & 0 \\
0 & 0 & 0 & 0 & 0 & 0 & 0 & 1 & 0 & 0 & 0 & 0 & 0 & 0 & 0 & 0 \\
1 & 0 & 0 & 0 & 0 & 0 & 0 & 0 & 0 & 0 & 0 & 0 & 0 & 0 & 0 & 0 \\
0 & 1 & 0 & 0 & 0 & 0 & 0 & 0 & 0 & 0 & 0 & 0 & 0 & 0 & 0 & 0 \\
0 & 0 & 1 & 0 & 0 & 0 & 0 & 0 & 0 & 0 & 0 & 0 & 0 & 0 & 0 & 0 \\
0 & 0 & 0 & 1 & 0 & 0 & 0 & 0 & 0 & 0 & 0 & 0 & 0 & 0 & 0 & 0 \\
0 & 0 & 0 & 0 & 0 & 0 & 0 & 0 & 0 & 0 & 0 & 0 & 1 & 0 & 0 & 0 \\
0 & 0 & 0 & 0 & 0 & 0 & 0 & 0 & 0 & 0 & 0 & 0 & 0 & 1 & 0 & 0 \\
0 & 0 & 0 & 0 & 0 & 0 & 0 & 0 & 0 & 0 & 0 & 0 & 0 & 0 & 1 & 0 \\
0 & 0 & 0 & 0 & 0 & 0 & 0 & 0 & 0 & 0 & 0 & 0 & 0 & 0 & 0 & 1 \\
0 & 0 & 0 & 0 & 0 & 0 & 0 & 0 & 1 & 0 & 0 & 0 & 0 & 0 & 0 & 0 \\
0 & 0 & 0 & 0 & 0 & 0 & 0 & 0 & 0 & 1 & 0 & 0 & 0 & 0 & 0 & 0 \\
0 & 0 & 0 & 0 & 0 & 0 & 0 & 0 & 0 & 0 & 1 & 0 & 0 & 0 & 0 & 0 \\
0 & 0 & 0 & 0 & 0 & 0 & 0 & 0 & 0 & 0 & 0 & 1 & 0 & 0 & 0 & 0
\end{array}\right),
$$




$$
B_{4}^{(16)}=\left(\begin{array}{cccccccccccccccc}
0 & 0 & 0 & 0 & 0 & 0 & 0 & 0 & 1 & 0 & 0 & 0 & 0 & 0 & 0 & 0 \\
0 & 0 & 0 & 0 & 0 & 0 & 0 & 0 & 0 & 1 & 0 & 0 & 0 & 0 & 0 & 0 \\
0 & 0 & 0 & 0 & 0 & 0 & 0 & 0 & 0 & 0 & 1 & 0 & 0 & 0 & 0 & 0 \\
0 & 0 & 0 & 0 & 0 & 0 & 0 & 0 & 0 & 0 & 0 & 1 & 0 & 0 & 0 & 0 \\
0 & 0 & 0 & 0 & 0 & 0 & 0 & 0 & 0 & 0 & 0 & 0 & 1 & 0 & 0 & 0 \\
0 & 0 & 0 & 0 & 0 & 0 & 0 & 0 & 0 & 0 & 0 & 0 & 0 & 1 & 0 & 0 \\
0 & 0 & 0 & 0 & 0 & 0 & 0 & 0 & 0 & 0 & 0 & 0 & 0 & 0 & 1 & 0 \\
0 & 0 & 0 & 0 & 0 & 0 & 0 & 0 & 0 & 0 & 0 & 0 & 0 & 0 & 0 & 1 \\
1 & 0 & 0 & 0 & 0 & 0 & 0 & 0 & 0 & 0 & 0 & 0 & 0 & 0 & 0 & 0 \\
0 & 1 & 0 & 0 & 0 & 0 & 0 & 0 & 0 & 0 & 0 & 0 & 0 & 0 & 0 & 0 \\
0 & 0 & 1 & 0 & 0 & 0 & 0 & 0 & 0 & 0 & 0 & 0 & 0 & 0 & 0 & 0 \\
0 & 0 & 0 & 1 & 0 & 0 & 0 & 0 & 0 & 0 & 0 & 0 & 0 & 0 & 0 & 0 \\
0 & 0 & 0 & 0 & 1 & 0 & 0 & 0 & 0 & 0 & 0 & 0 & 0 & 0 & 0 & 0 \\
0 & 0 & 0 & 0 & 0 & 1 & 0 & 0 & 0 & 0 & 0 & 0 & 0 & 0 & 0 & 0 \\
0 & 0 & 0 & 0 & 0 & 0 & 1 & 0 & 0 & 0 & 0 & 0 & 0 & 0 & 0 & 0 \\
0 & 0 & 0 & 0 & 0 & 0 & 0 & 1 & 0 & 0 & 0 & 0 & 0 & 0 & 0 & 0
\end{array}\right),
$$

$$
X^{(16)}=\left(\begin{array}{cccccccccccccccc}
1 & 0 & 0 & 0 & 0 & 0 & 0 & 0 & 0 & 0 & 0 & 0 & 0 & 0 & 0 & 0 \\
0 & 0 & 1 & 0 & 0 & 0 & 0 & 0 & 0 & 0 & 0 & 0 & 0 & 0 & 0 & 0 \\
0 & 1 & 0 & 0 & 0 & 0 & 0 & 0 & 0 & 0 & 0 & 0 & 0 & 0 & 0 & 0 \\
0 & 0 & 0 & 1 & 0 & 0 & 0 & 0 & 0 & 0 & 0 & 0 & 0 & 0 & 0 & 0 \\
0 & 0 & 0 & 0 & 0 & 0 & 0 & 0 & 1 & 0 & 0 & 0 & 0 & 0 & 0 & 0 \\
0 & 0 & 0 & 0 & 0 & 0 & 0 & 0 & 0 & 0 & 1 & 0 & 0 & 0 & 0 & 0 \\
0 & 0 & 0 & 0 & 0 & 0 & 0 & 0 & 0 & 1 & 0 & 0 & 0 & 0 & 0 & 0 \\
0 & 0 & 0 & 0 & 0 & 0 & 0 & 0 & 0 & 0 & 0 & 1 & 0 & 0 & 0 & 0 \\
0 & 0 & 0 & 0 & 1 & 0 & 0 & 0 & 0 & 0 & 0 & 0 & 0 & 0 & 0 & 0 \\
0 & 0 & 0 & 0 & 0 & 0 & 1 & 0 & 0 & 0 & 0 & 0 & 0 & 0 & 0 & 0 \\
0 & 0 & 0 & 0 & 0 & 1 & 0 & 0 & 0 & 0 & 0 & 0 & 0 & 0 & 0 & 0 \\
0 & 0 & 0 & 0 & 0 & 0 & 0 & 1 & 0 & 0 & 0 & 0 & 0 & 0 & 0 & 0 \\
0 & 0 & 0 & 0 & 0 & 0 & 0 & 0 & 0 & 0 & 0 & 0 & 1 & 0 & 0 & 0 \\
0 & 0 & 0 & 0 & 0 & 0 & 0 & 0 & 0 & 0 & 0 & 0 & 0 & 0 & 1 & 0 \\
0 & 0 & 0 & 0 & 0 & 0 & 0 & 0 & 0 & 0 & 0 & 0 & 0 & 1 & 0 & 0 \\
0 & 0 & 0 & 0 & 0 & 0 & 0 & 0 & 0 & 0 & 0 & 0 & 0 & 0 & 0 & 1
\end{array}\right),
$$




$$
Y^{(16)}=\left(\begin{array}{cccccccccccccccc}
1 & 0 & 0 & 0 & 0 & 0 & 0 & 0 & 0 & 0 & 0 & 0 & 0 & 0 & 0 & 0 \\
0 & 1 & 0 & 0 & 0 & 0 & 0 & 0 & 0 & 0 & 0 & 0 & 0 & 0 & 0 & 0 \\
0 & 0 & 0 & 0 & 0 & 0 & 0 & 0 & 1 & 0 & 0 & 0 & 0 & 0 & 0 & 0 \\
0 & 0 & 0 & 0 & 0 & 0 & 0 & 0 & 0 & 1 & 0 & 0 & 0 & 0 & 0 & 0 \\
0 & 0 & 1 & 0 & 0 & 0 & 0 & 0 & 0 & 0 & 0 & 0 & 0 & 0 & 0 & 0 \\
0 & 0 & 0 & 1 & 0 & 0 & 0 & 0 & 0 & 0 & 0 & 0 & 0 & 0 & 0 & 0 \\
0 & 0 & 0 & 0 & 0 & 0 & 0 & 0 & 0 & 0 & 1 & 0 & 0 & 0 & 0 & 0 \\
0 & 0 & 0 & 0 & 0 & 0 & 0 & 0 & 0 & 0 & 0 & 1 & 0 & 0 & 0 & 0 \\
0 & 0 & 0 & 0 & 1 & 0 & 0 & 0 & 0 & 0 & 0 & 0 & 0 & 0 & 0 & 0 \\
0 & 0 & 0 & 0 & 0 & 1 & 0 & 0 & 0 & 0 & 0 & 0 & 0 & 0 & 0 & 0 \\
0 & 0 & 0 & 0 & 0 & 0 & 0 & 0 & 0 & 0 & 0 & 0 & 1 & 0 & 0 & 0 \\
0 & 0 & 0 & 0 & 0 & 0 & 0 & 0 & 0 & 0 & 0 & 0 & 0 & 1 & 0 & 0 \\
0 & 0 & 0 & 0 & 0 & 0 & 1 & 0 & 0 & 0 & 0 & 0 & 0 & 0 & 0 & 0 \\
0 & 0 & 0 & 0 & 0 & 0 & 0 & 1 & 0 & 0 & 0 & 0 & 0 & 0 & 0 & 0 \\
0 & 0 & 0 & 0 & 0 & 0 & 0 & 0 & 0 & 0 & 0 & 0 & 0 & 0 & 1 & 0 \\
0 & 0 & 0 & 0 & 0 & 0 & 0 & 0 & 0 & 0 & 0 & 0 & 0 & 0 & 0 & 1
\end{array}\right) .
$$

The generators for $16^{\prime}$ and $16^{\prime \prime}$ are the same except $Y^{\left(16^{\prime}\right)}=\omega Y^{(16)}$ and $Y^{\left(16^{\prime \prime}\right)}=\omega^{2} Y^{(16)}$. The generators for 48 are

$$
\begin{aligned}
& A_{1}^{(48)}=\left(\begin{array}{ccc}
A_{1}^{(16)} & 0 & 0 \\
0 & A_{1}^{(16)} & 0 \\
0 & 0 & A_{1}^{(16)}
\end{array}\right), \quad B_{1}^{(48)}=\left(\begin{array}{ccc}
B_{1}^{(16)} & 0 & 0 \\
0 & B_{1}^{(16)} & 0 \\
0 & 0 & B_{1}^{(16)}
\end{array}\right), \\
& A_{2}^{(48)}=\left(\begin{array}{ccc}
A_{2}^{(16)} & 0 & 0 \\
0 & A_{4}^{(16)} & 0 \\
0 & 0 & A_{3}^{(16)}
\end{array}\right), \quad B_{2}^{(48)}=\left(\begin{array}{ccc}
B_{2}^{(16)} & 0 & 0 \\
0 & B_{4}^{(16)} & 0 \\
0 & 0 & B_{3}^{(16)}
\end{array}\right), \\
& A_{3}^{(48)}=\left(\begin{array}{ccc}
A_{3}^{(16)} & 0 & 0 \\
0 & A_{2}^{(16)} & 0 \\
0 & 0 & A_{4}^{(16)}
\end{array}\right), \quad B_{3}^{(48)}=\left(\begin{array}{ccc}
B_{3}^{(16)} & 0 & 0 \\
0 & B_{2}^{(16)} & 0 \\
0 & 0 & B_{4}^{(16)}
\end{array}\right), \\
& A_{4}^{(48)}=\left(\begin{array}{ccc}
A_{4}^{(16)} & 0 & 0 \\
0 & A_{3}^{(16)} & 0 \\
0 & 0 & A_{2}^{(16)}
\end{array}\right), \quad B_{4}^{(48)}=\left(\begin{array}{ccc}
B_{4}^{(16)} & 0 & 0 \\
0 & B_{3}^{(16)} & 0 \\
0 & 0 & B_{2}^{(16)}
\end{array}\right), \\
& X^{(48)}=\left(\begin{array}{ccc}
X^{(16)} & 0 & 0 \\
0 & -X^{(16)} Y^{(16)} X^{(16)}\left(Y^{(16)}\right)^{2} & 0 \\
0 & 0 & -Y^{(16)} X^{(16)}\left(Y^{(16)}\right)^{2}
\end{array}\right), \quad Y^{(48)}=\left(\begin{array}{ccc}
0 & 0 & I \\
I & 0 & 0 \\
0 & I & 0
\end{array}\right) .
\end{aligned}
$$


And the generators for 8 are

$$
\begin{aligned}
& A_{1}^{(8)}=\operatorname{diag}\left(\omega, \omega^{2}, 1,1,1,1,1,1\right), \quad A_{2}^{(8)}=\operatorname{diag}\left(1,1, \omega, \omega^{2}, 1,1,1,1\right), \\
& A_{3}^{(8)}=\operatorname{diag}\left(1,1,1,1, \omega, \omega^{2}, 1,1\right), \quad A_{4}^{(8)}=\operatorname{diag}\left(1,1,1,1,1,1, \omega, \omega^{2}\right), \\
& B_{1}^{(8)}=\left(\begin{array}{cccccccc}
0 & 1 & 0 & 0 & 0 & 0 & 0 & 0 \\
1 & 0 & 0 & 0 & 0 & 0 & 0 & 0 \\
0 & 0 & 1 & 0 & 0 & 0 & 0 & 0 \\
0 & 0 & 0 & 1 & 0 & 0 & 0 & 0 \\
0 & 0 & 0 & 0 & 1 & 0 & 0 & 0 \\
0 & 0 & 0 & 0 & 0 & 1 & 0 & 0 \\
0 & 0 & 0 & 0 & 0 & 0 & 1 & 0 \\
0 & 0 & 0 & 0 & 0 & 0 & 0 & 1
\end{array}\right), \quad B_{2}^{(8)}=\left(\begin{array}{cccccccc}
1 & 0 & 0 & 0 & 0 & 0 & 0 & 0 \\
0 & 1 & 0 & 0 & 0 & 0 & 0 & 0 \\
0 & 0 & 0 & 1 & 0 & 0 & 0 & 0 \\
0 & 0 & 1 & 0 & 0 & 0 & 0 & 0 \\
0 & 0 & 0 & 0 & 1 & 0 & 0 & 0 \\
0 & 0 & 0 & 0 & 0 & 1 & 0 & 0 \\
0 & 0 & 0 & 0 & 0 & 0 & 1 & 0 \\
0 & 0 & 0 & 0 & 0 & 0 & 0 & 1
\end{array}\right), \\
& B_{3}^{(8)}=\left(\begin{array}{cccccccc}
1 & 0 & 0 & 0 & 0 & 0 & 0 & 0 \\
0 & 1 & 0 & 0 & 0 & 0 & 0 & 0 \\
0 & 0 & 1 & 0 & 0 & 0 & 0 & 0 \\
0 & 0 & 0 & 1 & 0 & 0 & 0 & 0 \\
0 & 0 & 0 & 0 & 0 & 1 & 0 & 0 \\
0 & 0 & 0 & 0 & 1 & 0 & 0 & 0 \\
0 & 0 & 0 & 0 & 0 & 0 & 1 & 0 \\
0 & 0 & 0 & 0 & 0 & 0 & 0 & 1
\end{array}\right), \quad B_{4}^{(8)}=\left(\begin{array}{cccccccc}
1 & 0 & 0 & 0 & 0 & 0 & 0 & 0 \\
0 & 1 & 0 & 0 & 0 & 0 & 0 & 0 \\
0 & 0 & 1 & 0 & 0 & 0 & 0 & 0 \\
0 & 0 & 0 & 1 & 0 & 0 & 0 & 0 \\
0 & 0 & 0 & 0 & 1 & 0 & 0 & 0 \\
0 & 0 & 0 & 0 & 0 & 1 & 0 & 0 \\
0 & 0 & 0 & 0 & 0 & 0 & 0 & 1 \\
0 & 0 & 0 & 0 & 0 & 0 & 1 & 0
\end{array}\right), \\
& X^{(8)}=\left(\begin{array}{cccccccc}
0 & 0 & 1 & 0 & 0 & 0 & 0 & 0 \\
0 & 0 & 0 & 1 & 0 & 0 & 0 & 0 \\
1 & 0 & 0 & 0 & 0 & 0 & 0 & 0 \\
0 & 1 & 0 & 0 & 0 & 0 & 0 & 0 \\
0 & 0 & 0 & 0 & 0 & 0 & 1 & 0 \\
0 & 0 & 0 & 0 & 0 & 0 & 0 & 1 \\
0 & 0 & 0 & 0 & 1 & 0 & 0 & 0 \\
0 & 0 & 0 & 0 & 0 & 1 & 0 & 0
\end{array}\right), \quad Y^{(8)}=\left(\begin{array}{cccccccc}
1 & 0 & 0 & 0 & 0 & 0 & 0 & 0 \\
0 & 1 & 0 & 0 & 0 & 0 & 0 & 0 \\
0 & 0 & 0 & 0 & 0 & 0 & 1 & 0 \\
0 & 0 & 0 & 0 & 0 & 0 & 0 & 1 \\
0 & 0 & 1 & 0 & 0 & 0 & 0 & 0 \\
0 & 0 & 0 & 1 & 0 & 0 & 0 & 0 \\
0 & 0 & 0 & 0 & 1 & 0 & 0 & 0 \\
0 & 0 & 0 & 0 & 0 & 1 & 0 & 0
\end{array}\right) .
\end{aligned}
$$

The generators for 6 are

$$
A_{1}=A_{2}=A_{3}=A_{4}=I \text {, }
$$


where $I$ is the identity matrix,

$$
\begin{array}{ll}
B_{1}^{(6)}=\operatorname{diag}(1,-1,1,-1,1,-1), & B_{2}^{(6)}=\operatorname{diag}(1,-1,-1,1,-1,1), \\
B_{3}^{(6)}=\operatorname{diag}(-1,1,1,-1,-1,1), & B_{4}^{(6)}=\operatorname{diag}(-1,1,-1,1,1,-1), \\
X^{(6)}=\left(\begin{array}{llllll}
1 & 0 & 0 & 0 & 0 & 0 \\
0 & 1 & 0 & 0 & 0 & 0 \\
0 & 0 & 0 & 1 & 0 & 0 \\
0 & 0 & 1 & 0 & 0 & 0 \\
0 & 0 & 0 & 0 & 0 & 1 \\
0 & 0 & 0 & 0 & 1 & 0
\end{array}\right), & Y^{(6)}=\left(\begin{array}{llllll}
0 & 0 & 0 & 0 & 1 & 0 \\
0 & 0 & 0 & 0 & 0 & 1 \\
1 & 0 & 0 & 0 & 0 & 0 \\
0 & 1 & 0 & 0 & 0 & 0 \\
0 & 0 & 1 & 0 & 0 & 0 \\
0 & 0 & 0 & 1 & 0 & 0
\end{array}\right) .
\end{array}
$$

It can be checked directly that each set of matrices respects Eqs. (4)-(7).

\section{Appendix B}

In this appendix, we give the symmetry invariants which are used in our model. These can be computed directly from the matrices given in Appendix A.

(i) $16 \times 16$ invariant $\left(x_{i}, x_{j}^{\prime} \sim 16\right)$ :

$$
\begin{aligned}
f_{1}\left(x_{i}, x_{j}^{\prime}\right) & =x_{1} x_{16}^{\prime}+x_{2} x_{15}^{\prime}+x_{3} x_{14}^{\prime}+x_{4} x_{13}^{\prime}+x_{5} x_{12}^{\prime}+x_{6} x_{11}^{\prime}+x_{7} x_{10}^{\prime}+x_{8} x_{9}^{\prime} \\
& +x_{9} x_{8}^{\prime}+x_{10} x_{7}^{\prime}+x_{11} x_{6}^{\prime}+x_{12} x_{5}^{\prime}+x_{13} x_{4}^{\prime}+x_{14} x_{3}^{\prime}+x_{15} x_{2}^{\prime}+x_{16} x_{1}^{\prime}
\end{aligned}
$$

(ii) $3 \times 3$ invariant $\left(t_{i}, t_{j}^{\prime} \sim 3\right)$ :

$$
f_{2}\left(t_{i}, t_{j}^{\prime}\right)=t_{1} t_{1}^{\prime}+t_{2} t_{2}^{\prime}+t_{3} t_{3}^{\prime}
$$

(iii) $48 \times 48$ invariant $\left(y_{i}, y_{j}^{\prime} \sim 48\right)$ :

$$
\begin{aligned}
f_{3}\left(y_{i}, y_{j}^{\prime}\right) & =y_{1} y_{16}^{\prime}+y_{2} y_{15}^{\prime}+y_{3} y_{14}^{\prime}+y_{4} y_{13}^{\prime}+y_{5} y_{12}^{\prime}+y_{6} y_{11}^{\prime}+y_{7} y_{10}^{\prime}+y_{8} y_{9}^{\prime} \\
& +y_{9} y_{8}^{\prime}+y_{10} y_{7}^{\prime}+y_{11} y_{6}^{\prime}+y_{12} y_{5}^{\prime}+y_{13} y_{4}^{\prime}+y_{14} y_{3}^{\prime}+y_{15} y_{2}^{\prime}+y_{16} y_{1}^{\prime}+y_{17} y_{32}^{\prime}+y_{18} y_{31}^{\prime} \\
& +y_{19} y_{30}^{\prime}+y_{20} y_{29}^{\prime}+y_{21} y_{28}^{\prime}+y_{22} y_{27}^{\prime}+y_{23} y_{26}^{\prime}+y_{24} y_{25}^{\prime}+y_{25} y_{24}^{\prime}+y_{26} y_{23}^{\prime}+y_{27} y_{22}^{\prime}+y_{28} y_{21}^{\prime} \\
& +y_{29} y_{20}^{\prime}+y_{30} y_{19}^{\prime}+y_{31} y_{18}^{\prime}+y_{32} y_{17}^{\prime}+y_{33} y_{48}^{\prime}+y_{34} y_{47}^{\prime}+y_{35} y_{46}^{\prime}+y_{36} y_{45}^{\prime}+y_{37} y_{44}^{\prime}+y_{38} y_{43}^{\prime} \\
& +y_{39} y_{42}^{\prime}+y_{40} y_{41}^{\prime}+y_{41} y_{40}^{\prime}+y_{42} y_{39}^{\prime}+y_{43} y_{38}^{\prime}+y_{44} y_{37}^{\prime}+y_{45} y_{36}^{\prime}+y_{46} y_{35}^{\prime}+y_{47} y_{34}^{\prime}+y_{48} y_{33}^{\prime}
\end{aligned}
$$

(iv) $8 \times 8$ invariant $\left(z_{i}, z_{j}^{\prime} \sim 8\right)$ :

$$
f_{4}\left(z_{i}, z_{j}^{\prime}\right)=z_{1} z_{2}^{\prime}+z_{2} z_{1}^{\prime}+z_{3} z_{4}^{\prime}+z_{4} z_{3}^{\prime}+z_{5} z_{6}^{\prime}+z_{6} z_{5}^{\prime}+z_{7} z_{8}^{\prime}+z_{8} z_{7}^{\prime}
$$


(v) $6 \times 6$ invariant $\left(w_{i}, w_{j}^{\prime} \sim 6\right)$ :

$$
f_{5}\left(w_{i}, w_{j}^{\prime}\right)=w_{1} w_{1}^{\prime}+w_{2} w_{2}^{\prime}+w_{3} w_{3}^{\prime}+w_{4} w_{4}^{\prime}+w_{5} w_{5}^{\prime}+w_{6} w_{6}^{\prime}
$$

(vi) $16 \times 16 \times 16$ invariant $\left(x_{i}, x_{j}^{\prime}, x_{k}^{\prime \prime} \sim 16\right)$ :

$$
\begin{aligned}
g_{1}\left(x_{i}, x_{j}^{\prime}, x_{k}^{\prime \prime}\right) & =x_{1} x_{1}^{\prime} x_{1}^{\prime \prime}+x_{2} x_{2}^{\prime} x_{2}^{\prime \prime}+x_{3} x_{3}^{\prime} x_{3}^{\prime \prime}+x_{4} x_{4}^{\prime} x_{4}^{\prime \prime}+x_{5} x_{5}^{\prime} x_{5}^{\prime \prime}+x_{6} x_{6}^{\prime} x_{6}^{\prime \prime}+x_{7} x_{7}^{\prime} x_{7}^{\prime \prime}+x_{8} x_{8}^{\prime} x_{8}^{\prime \prime} \\
& +x_{9} x_{9}^{\prime} x_{9}^{\prime \prime}+x_{10} x_{10}^{\prime} x_{10}^{\prime \prime}+x_{11} x_{11}^{\prime} x_{11}^{\prime \prime}+x_{12} x_{12}^{\prime} x_{12}^{\prime \prime}+x_{13} x_{13}^{\prime} x_{13}^{\prime \prime}+x_{14} x_{14}^{\prime} x_{14}^{\prime \prime}+x_{15} x_{15}^{\prime} x_{15}^{\prime \prime} \\
& +x_{16} x_{16}^{\prime} x_{16}^{\prime \prime}
\end{aligned}
$$

(vii) $3 \times 16 \times 48$ invariant $\left(t_{i} \sim 3, x_{j} \sim 16, y_{k} \sim 48\right)$ :

$$
\begin{aligned}
g_{2}\left(t_{i}, x_{j}, y_{k}\right) & =t_{1}\left(x_{16} y_{33}+x_{15} y_{34}+x_{8} y_{35}+x_{7} y_{36}+x_{14} y_{37}+x_{13} y_{38}+x_{6} y_{39}+x_{5} y_{40}\right. \\
& \left.+x_{12} y_{41}+x_{11} y_{42}+x_{4} y_{43}+x_{3} y_{44}+x_{10} y_{45}+x_{9} y_{46}+x_{2} y_{47}+x_{1} y_{48}\right) \\
& +t_{2}\left(x_{16} y_{1}+x_{7} y_{10}+x_{6} y_{11}+x_{5} y_{12}+x_{4} y_{13}+x_{3} y_{14}+x_{2} y_{15}+x_{1} y_{16}\right. \\
& \left.+x_{15} y_{2}+x_{14} y_{3}+x_{13} y_{4}+x_{12} y_{5}+x_{11} y_{6}+x_{10} y_{7}+x_{9} y_{8}+x_{8} y_{9}\right) \\
& +t_{3}\left(x_{16} y_{17}+x_{15} y_{18}+x_{12} y_{19}+x_{11} y_{20}+x_{8} y_{21}+x_{7} y_{22}+x_{4} y_{23}+x_{3} y_{24}\right. \\
& \left.+x_{14} y_{25}+x_{13} y_{26}+x_{10} y_{27}+x_{9} y_{28}+x_{6} y_{29}+x_{5} y_{30}+x_{2} y_{31}+x_{1} y_{32}\right)
\end{aligned}
$$


(viii) $8 \times 16 \times 48$ invariant $\left(z_{i} \sim 8, x_{j} \sim 16, y_{k} \sim 48\right)$ :

$$
\begin{aligned}
& g_{3}\left(z_{i}, x_{k}, y_{j}\right)=z_{1}\left(x_{15} y_{1}+x_{5} y_{11}+x_{3} y_{13}+x_{1} y_{15}+x_{15} y_{17}+x_{11} y_{19}+x_{7} y_{21}+x_{3} y_{23}\right. \\
& +x_{13} y_{25}+x_{9} y_{27}+x_{5} y_{29}+x_{13} y_{3}+x_{1} y_{31}+x_{15} y_{33}+x_{7} y_{35}+x_{13} y_{37} \\
& \left.+x_{5} y_{39}+x_{11} y_{41}+x_{3} y_{43}+x_{9} y_{45}+x_{1} y_{47}+x_{11} y_{5}+x_{9} y_{7}+x_{7} y_{9}\right) \\
& +z_{2}\left(x_{8} y_{10}+x_{6} y_{12}+x_{4} y_{14}+x_{2} y_{16}+x_{16} y_{18}+x_{16} y_{2}+x_{12} y_{20}+x_{8} y_{22}\right. \\
& +x_{4} y_{24}+x_{14} y_{26}+x_{10} y_{28}+x_{6} y_{30}+x_{2} y_{32}+x_{16} y_{34}+x_{8} y_{36}+x_{14} y_{38} \\
& \left.+x_{14} y_{4}+x_{6} y_{40}+x_{12} y_{42}+x_{4} y_{44}+x_{10} y_{46}+x_{2} y_{48}+x_{12} y_{6}+x_{10} y_{8}\right) \\
& +z_{3}\left(x_{14} y_{1}+x_{5} y_{10}+x_{2} y_{13}+x_{1} y_{14}-x_{14} y_{17}-x_{13} y_{18}-x_{10} y_{19}+x_{13} y_{2}\right. \\
& -x_{9} y_{20}-x_{6} y_{21}-x_{5} y_{22}-x_{2} y_{23}-x_{1} y_{24}-x_{14} y_{33}-x_{13} y_{34}-x_{6} y_{35} \\
& \left.-x_{5} y_{36}-x_{10} y_{41}-x_{9} y_{42}-x_{2} y_{43}-x_{1} y_{44}+x_{10} y_{5}+x_{9} y_{6}+x_{6} y_{9}\right) \\
& +z_{4}\left(x_{8} y_{11}+x_{7} y_{12}+x_{4} y_{15}+x_{3} y_{16}-x_{16} y_{25}-x_{15} y_{26}-x_{12} y_{27}-x_{11} y_{28}\right. \\
& -x_{8} y_{29}+x_{16} y_{3}-x_{7} y_{30}-x_{4} y_{31}-x_{3} y_{32}-x_{16} y_{37}-x_{15} y_{38}-x_{8} y_{39}+x_{15} y_{4} \\
& \left.-x_{7} y_{40}-x_{12} y_{45}-x_{11} y_{46}-x_{4} y_{47}-x_{3} y_{48}+x_{12} y_{7}+x_{11} y_{8}\right) \\
& +z_{5}\left(-x_{12} y_{1}-x_{3} y_{10}-x_{2} y_{11}-x_{1} y_{12}+x_{12} y_{17}+x_{11} y_{18}-x_{11} y_{2}+x_{4} y_{21}+x_{3} y_{22}\right. \\
& +x_{10} y_{25}+x_{9} y_{26}+x_{2} y_{29}-x_{10} y_{3}+x_{1} y_{30}-x_{12} y_{33}-x_{11} y_{34}-x_{4} y_{35}-x_{3} y_{36} \\
& \left.-x_{10} y_{37}-x_{9} y_{38}-x_{2} y_{39}-x_{9} y_{4}-x_{1} y_{40}-x_{4} y_{9}\right)+z_{6}\left(-x_{8} y_{13}-x_{7} y_{14}-x_{6} y_{15}\right. \\
& -x_{5} y_{16}+x_{16} y_{19}+x_{15} y_{20}+x_{8} y_{23}+x_{7} y_{24}+x_{14} y_{27}+x_{13} y_{28}+x_{6} y_{31}+x_{5} y_{32} \\
& -x_{16} y_{41}-x_{15} y_{42}-x_{8} y_{43}-x_{7} y_{44}-x_{14} y_{45}-x_{13} y_{46}-x_{6} y_{47}-x_{5} y_{48}-x_{16} y_{5} \\
& \left.-x_{15} y_{6}-x_{14} y_{7}-x_{13} y_{8}\right)+z_{7}\left(-x_{8} y_{1}-x_{8} y_{17}-x_{7} y_{18}-x_{4} y_{19}-x_{7} y_{2}-x_{3} y_{20}\right. \\
& -x_{6} y_{25}-x_{5} y_{26}-x_{2} y_{27}-x_{1} y_{28}-x_{6} y_{3}+x_{8} y_{33}+x_{7} y_{34}+x_{6} y_{37}+x_{5} y_{38}-x_{5} y_{4} \\
& \left.+x_{4} y_{41}+x_{3} y_{42}+x_{2} y_{45}+x_{1} y_{46}-x_{4} y_{5}-x_{3} y_{6}-x_{2} y_{7}-x_{1} y_{8}\right) \\
& +z_{8}\left(x_{15} y_{36}-x_{14} y_{11}-x_{13} y_{12}-x_{12} y_{13}-x_{11} y_{14}-x_{10} y_{15}-x_{9} y_{16}-x_{16} y_{21}\right. \\
& -x_{15} y_{22}-x_{12} y_{23}-x_{11} y_{24}-x_{14} y_{29}-x_{13} y_{30}-x_{10} y_{31}-x_{9} y_{32} \\
& \left.+x_{16} y_{35}-x_{15} y_{10}+x_{14} y_{39}+x_{13} y_{40}+x_{12} y_{43}+x_{11} y_{44}+x_{10} y_{47}+x_{9} y_{48}-x_{16} y_{9}\right)
\end{aligned}
$$

(ix) $16 \times 48 \times 48$ invariant $\left(x_{i} \sim 16 ; y_{j}, y_{k}^{\prime} \sim 48\right)$ :

$$
\begin{aligned}
g_{4}\left(x_{i}, y_{j}, y_{k}^{\prime}\right) & =x_{1} y_{1} y_{1}^{\prime}+x_{2} y_{2} y_{2}^{\prime}+x_{3} y_{3} y_{3}^{\prime}+x_{4} y_{4} y_{4}^{\prime}+x_{5} y_{5} y_{5}^{\prime}+x_{6} y_{6} y_{6}^{\prime}+x_{7} y_{7} y_{7}^{\prime}+x_{8} y_{8} y_{8}^{\prime} \\
& +x_{9} y_{9} y_{9}^{\prime}+x_{10} y_{10} y_{10}^{\prime}+x_{11} y_{11} y_{11}^{\prime}+x_{12} y_{12} y_{12}^{\prime}+x_{13} y_{13} y_{13}^{\prime}+x_{14} y_{14} y_{14}^{\prime}+x_{15} y_{15} y_{15}^{\prime} \\
& +x_{16} y_{16} y_{16}^{\prime}+x_{1} y_{17} y_{17}^{\prime}+x_{2} y_{18} y_{18}^{\prime}+x_{3} y_{25} y_{25}^{\prime}+x_{4} y_{26} y_{26}^{\prime}+x_{5} y_{19} y_{19}^{\prime}+x_{6} y_{20} y_{20}^{\prime}+x_{7} y_{27} y_{27}^{\prime} \\
& +x_{8} y_{28} y_{28}^{\prime}+x_{9} y_{21} y_{21}^{\prime}+x_{10} y_{22} y_{22}^{\prime}+x_{11} y_{29} y_{29}^{\prime}+x_{12} y_{30} y_{30}^{\prime}+x_{13} y_{23} y_{23}^{\prime}+x_{14} y_{24} y_{24}^{\prime} \\
& +x_{15} y_{31} y_{31}^{\prime}+x_{16} y_{32} y_{32}^{\prime}+x_{1} y_{33} y_{33}^{\prime}+x_{2} y_{34} y_{34}^{\prime}+x_{3} y_{37} y_{37}^{\prime}+x_{4} y_{38} y_{38}^{\prime}+x_{5} y_{41} y_{41}^{\prime} \\
& +x_{6} y_{42} y_{42}^{\prime}+x_{7} y_{45} y_{45}^{\prime}+x_{8} y_{46} y_{46}^{\prime}+x_{9} y_{35} y_{35}^{\prime}+x_{10} y_{36} y_{36}^{\prime}+x_{11} y_{39} y_{39}^{\prime}+x_{12} y_{40} y_{40}^{\prime} \\
& +x_{13} y_{43} y_{43}^{\prime}+x_{14} y_{44} y_{44}^{\prime}+x_{15} y_{47} y_{47}^{\prime}+x_{16} y_{48} y_{48}^{\prime}
\end{aligned}
$$


(x) $\mathbf{1}^{\prime} \times 3 \times 3$ invariant $\left(s^{\prime} \sim 1^{\prime} ; t_{i}, t_{j}^{\prime} \sim 3\right)$ :

$$
g_{5}\left(s^{\prime}, t_{i}, t_{j}^{\prime}\right)=s^{\prime}\left(t_{1} t_{1}^{\prime}+\omega^{2} t_{2} t_{2}^{\prime}+\omega t_{3} t_{3}^{\prime}\right)
$$

(xi) $1^{\prime \prime} \times 3 \times 3$ invariant $\left(s^{\prime \prime} \sim 1^{\prime \prime} ; t_{i}, t_{j}^{\prime} \sim 3\right)$ :

$$
g_{6}\left(s^{\prime \prime}, t_{i}, t_{j}\right)=s^{\prime \prime}\left(t_{1} t_{1}^{\prime}+\omega t_{2} t_{2}^{\prime}+\omega^{2} t_{3} t_{3}^{\prime}\right)
$$

(xii) $6 \times 16 \times 16$ invariant $\left(w_{i} \sim 6 ; x_{j}, x_{k}^{\prime} \sim 16\right)$ :

$$
\begin{aligned}
g_{7}\left(w_{i}, x_{j}, x_{k}^{\prime}\right) & =w_{1}\left(x_{1} x_{16}^{\prime}+x_{2} x_{15}^{\prime}+x_{3} x_{14}^{\prime}+x_{4} x_{13}^{\prime}-x_{5} x_{12}^{\prime}-x_{6} x_{11}^{\prime}-x_{7} x_{10}^{\prime}-x_{8} x_{9}^{\prime}\right. \\
& \left.-x_{9} x_{8}^{\prime}-x_{10} x_{7}^{\prime}-x_{11} x_{6}^{\prime}-x_{12} x_{5}^{\prime}+x_{13} x_{4}^{\prime}+x_{14} x_{3}^{\prime}+x_{15} x_{2}^{\prime}+x_{16} x_{1}^{\prime}\right) \\
& +w_{2}\left(x_{1} x_{16}^{\prime}-x_{2} x_{15}^{\prime}-x_{3} x_{14}^{\prime}+x_{4} x_{13}^{\prime}+x_{5} x_{12}^{\prime}-x_{6} x_{11}^{\prime}-x_{7} x_{10}^{\prime}+x_{8} x_{9}^{\prime}\right. \\
& \left.+x_{9} x_{8}^{\prime}-x_{10} x_{7}^{\prime}-x_{11} x_{6}^{\prime}+x_{12} x_{5}^{\prime}+x_{13} x_{4}^{\prime}-x_{14} x_{3}^{\prime}-x_{15} x_{2}^{\prime}+x_{16} x_{1}^{\prime}\right) \\
& +w_{3}\left(x_{1} x_{16}^{\prime}+x_{2} x_{15}^{\prime}-x_{3} x_{14}^{\prime}-x_{4} x_{13}^{\prime}+x_{5} x_{12}^{\prime}+x_{6} x_{11}^{\prime}-x_{7} x_{10}^{\prime}-x_{8} x_{9}^{\prime}\right. \\
& \left.-x_{9} x_{8}^{\prime}-x_{10} x_{7}^{\prime}+x_{11} x_{6}^{\prime}+x_{12} x_{5}^{\prime}-x_{13} x_{4}^{\prime}-x_{14} x_{3}^{\prime}+x_{15} x_{2}^{\prime}+x_{16} x_{1}^{\prime}\right) \\
& +w_{4}\left(x_{1} x_{16}^{\prime}-x_{2} x_{15}^{\prime}+x_{3} x_{14}^{\prime}-x_{4} x_{13}^{\prime}-x_{5} x_{12}^{\prime}+x_{6} x_{11}^{\prime}-x_{7} x_{10}^{\prime}+x_{8} x_{9}^{\prime}\right. \\
& \left.+x_{9} x_{8}^{\prime}-x_{10} x_{7}^{\prime}+x_{11} x_{6}^{\prime}-x_{12} x_{5}^{\prime}-x_{13} x_{4}^{\prime}+x_{14} x_{3}^{\prime}-x_{15} x_{2}^{\prime}+x_{16} x_{1}^{\prime}\right) \\
& +w_{5}\left(x_{1} x_{16}^{\prime}+x_{2} x_{15}^{\prime}-x_{3} x_{14}^{\prime}-x_{4} x_{13}^{\prime}-x_{5} x_{12}^{\prime}-x_{6} x_{11}^{\prime}+x_{7} x_{10}^{\prime}+x_{8} x_{9}^{\prime}\right. \\
& \left.+x_{9} x_{8}^{\prime}-x_{10} x_{7}^{\prime}-x_{11} x_{6}^{\prime}-x_{12} x_{5}^{\prime}-x_{13} x_{4}^{\prime}-x_{14} x_{3}^{\prime}+x_{15} x_{2}^{\prime}+x_{16} x_{1}^{\prime}\right) \\
& +w_{6}\left(x_{1} x_{16}^{\prime}-x_{2} x_{15}^{\prime}+x_{3} x_{14}^{\prime}-x_{4} x_{13}^{\prime}+x_{5} x_{12}^{\prime}-x_{6} x_{11}^{\prime}+x_{7} x_{10}^{\prime}-x_{8} x_{9}^{\prime}\right. \\
& \left.-x_{9} x_{8}^{\prime}+x_{10} x_{7}^{\prime}-x_{11} x_{6}^{\prime}+x_{12} x_{5}^{\prime}-x_{13} x_{4}^{\prime}+x_{14} x_{3}^{\prime}-x_{15} x_{2}^{\prime}+x_{16} x_{1}^{\prime}\right)
\end{aligned}
$$

(xiii) $6 \times 16 \times 16^{\prime}$ invariant $\left(w_{i} \sim 6 ; x_{j}, x_{k}^{\prime} \sim 16^{\prime}\right)$ :

$$
\begin{aligned}
g_{8}\left(w_{i}, x_{j}, x_{k}^{\prime}\right) & =w_{1}\left(x_{1} x_{16}^{\prime}+x_{2} x_{15}^{\prime}+x_{3} x_{14}^{\prime}+x_{4} x_{13}^{\prime}-x_{5} x_{12}^{\prime}-x_{6} x_{11}^{\prime}-x_{7} x_{10}^{\prime}-x_{8} x_{9}^{\prime}\right. \\
& \left.-x_{9} x_{8}^{\prime}-x_{10} x_{7}^{\prime}-x_{11} x_{6}^{\prime}-x_{12} x_{5}^{\prime}+x_{13} x_{4}^{\prime}+x_{14} x_{3}^{\prime}+x_{15} x_{2}^{\prime}+x_{16} x_{1}^{\prime}\right) \\
& +w_{2}\left(x_{1} x_{16}^{\prime}-x_{2} x_{15}^{\prime}-x_{3} x_{14}^{\prime}+x_{4} x_{13}^{\prime}+x_{5} x_{12}^{\prime}-x_{6} x_{11}^{\prime}-x_{7} x_{10}^{\prime}+x_{8} x_{9}^{\prime}\right. \\
& \left.+x_{9} x_{8}^{\prime}-x_{10} x_{7}^{\prime}-x_{11} x_{6}^{\prime}+x_{12} x_{5}^{\prime}+x_{13} x_{4}^{\prime}-x_{14} x_{3}^{\prime}-x_{15} x_{2}^{\prime}+x_{16} x_{1}^{\prime}\right) \\
& +\omega^{2} w_{3}\left(x_{1} x_{16}^{\prime}+x_{2} x_{15}^{\prime}-x_{3} x_{14}^{\prime}-x_{4} x_{13}^{\prime}+x_{5} x_{12}^{\prime}+x_{6} x_{11}^{\prime}-x_{7} x_{10}^{\prime}-x_{8} x_{9}^{\prime}\right. \\
& \left.-x_{9} x_{8}^{\prime}-x_{10} x_{7}^{\prime}+x_{11} x_{6}^{\prime}+x_{12} x_{5}^{\prime}-x_{13} x_{4}^{\prime}-x_{14} x_{3}^{\prime}+x_{15} x_{2}^{\prime}+x_{16} x_{1}^{\prime}\right) \\
& +\omega^{2} w_{4}\left(x_{1} x_{16}^{\prime}-x_{2} x_{15}^{\prime}+x_{3} x_{14}^{\prime}-x_{4} x_{13}^{\prime}-x_{5} x_{12}^{\prime}+x_{6} x_{11}^{\prime}-x_{7} x_{10}^{\prime}+x_{8} x_{9}^{\prime}\right. \\
& \left.+x_{9} x_{8}^{\prime}-x_{10} x_{7}^{\prime}+x_{11} x_{6}^{\prime}-x_{12} x_{5}^{\prime}-x_{13} x_{4}^{\prime}+x_{14} x_{3}^{\prime}-x_{15} x_{2}^{\prime}+x_{16} x_{1}^{\prime}\right) \\
& +\omega w_{5}\left(x_{1} x_{16}^{\prime}+x_{2} x_{15}^{\prime}-x_{3} x_{14}^{\prime}-x_{4} x_{13}^{\prime}-x_{5} x_{12}^{\prime}-x_{6} x_{11}^{\prime}+x_{7} x_{10}^{\prime}+x_{8} x_{9}^{\prime}\right. \\
& \left.+x_{9} x_{8}^{\prime}-x_{10} x_{7}^{\prime}-x_{11} x_{6}^{\prime}-x_{12} x_{5}^{\prime}-x_{13} x_{4}^{\prime}-x_{14} x_{3}^{\prime}+x_{15} x_{2}^{\prime}+x_{16} x_{1}^{\prime}\right) \\
& +\omega w_{6}\left(x_{1} x_{16}^{\prime}-x_{2} x_{15}^{\prime}+x_{3} x_{14}^{\prime}-x_{4} x_{13}^{\prime}+x_{5} x_{12}^{\prime}-x_{6} x_{11}^{\prime}+x_{7} x_{10}^{\prime}-x_{8} x_{9}^{\prime}\right. \\
& \left.-x_{9} x_{8}^{\prime}+x_{10} x_{7}^{\prime}-x_{11} x_{6}^{\prime}+x_{12} x_{5}^{\prime}-x_{13} x_{4}^{\prime}+x_{14} x_{3}^{\prime}-x_{15} x_{2}^{\prime}+x_{16} x_{1}^{\prime}\right)
\end{aligned}
$$


(xiv) $6 \times 16 \times 16^{\prime \prime}$ invariant $\left(w_{i} \sim 6 ; x_{j}, x_{k}^{\prime} \sim 16^{\prime \prime}\right)$ :

$$
\begin{aligned}
g_{9}\left(w_{i}, x_{j}, x_{k}^{\prime}\right) & =w_{1}\left(x_{1} x_{16}^{\prime}+x_{2} x_{15}^{\prime}+x_{3} x_{14}^{\prime}+x_{4} x_{13}^{\prime}-x_{5} x_{12}^{\prime}-x_{6} x_{11}^{\prime}-x_{7} x_{10}^{\prime}-x_{8} x_{9}^{\prime}\right. \\
& \left.-x_{9} x_{8}^{\prime}-x_{10} x_{7}^{\prime}-x_{11} x_{6}^{\prime}-x_{12} x_{5}^{\prime}+x_{13} x_{4}^{\prime}+x_{14} x_{3}^{\prime}+x_{15} x_{2}^{\prime}+x_{16} x_{1}^{\prime}\right) \\
& +w_{2}\left(x_{1} x_{16}^{\prime}-x_{2} x_{15}^{\prime}-x_{3} x_{14}^{\prime}+x_{4} x_{13}^{\prime}+x_{5} x_{12}^{\prime}-x_{6} x_{11}^{\prime}-x_{7} x_{10}^{\prime}+x_{8} x_{9}^{\prime}\right. \\
& \left.+x_{9} x_{8}^{\prime}-x_{10} x_{7}^{\prime}-x_{11} x_{6}^{\prime}+x_{12} x_{5}^{\prime}+x_{13} x_{4}^{\prime}-x_{14} x_{3}^{\prime}-x_{15} x_{2}^{\prime}+x_{16} x_{1}^{\prime}\right) \\
& +\omega w_{3}\left(x_{1} x_{16}^{\prime}+x_{2} x_{15}^{\prime}-x_{3} x_{14}^{\prime}-x_{4} x_{13}^{\prime}+x_{5} x_{12}^{\prime}+x_{6} x_{11}^{\prime}-x_{7} x_{10}^{\prime}-x_{8} x_{9}^{\prime}\right. \\
& \left.-x_{9} x_{8}^{\prime}-x_{10} x_{7}^{\prime}+x_{11} x_{6}^{\prime}+x_{12} x_{5}^{\prime}-x_{13} x_{4}^{\prime}-x_{14} x_{3}^{\prime}+x_{15} x_{2}^{\prime}+x_{16} x_{1}^{\prime}\right) \\
& +\omega w_{4}\left(x_{1} x_{16}^{\prime}-x_{2} x_{15}^{\prime}+x_{3} x_{14}^{\prime}-x_{4} x_{13}^{\prime}-x_{5} x_{12}^{\prime}+x_{6} x_{11}^{\prime}-x_{7} x_{10}^{\prime}+x_{8} x_{9}^{\prime}\right. \\
& \left.+x_{9} x_{8}^{\prime}-x_{10} x_{7}^{\prime}+x_{11} x_{6}^{\prime}-x_{12} x_{5}^{\prime}-x_{13} x_{4}^{\prime}+x_{14} x_{3}^{\prime}-x_{15} x_{2}^{\prime}+x_{16} x_{1}^{\prime}\right) \\
& +\omega^{2} w_{5}\left(x_{1} x_{16}^{\prime}+x_{2} x_{15}^{\prime}-x_{3} x_{14}^{\prime}-x_{4} x_{13}^{\prime}-x_{5} x_{12}^{\prime}-x_{6} x_{11}^{\prime}+x_{7} x_{10}^{\prime}+x_{8} x_{9}^{\prime}\right. \\
& \left.+x_{9} x_{8}^{\prime}-x_{10} x_{7}^{\prime}-x_{11} x_{6}^{\prime}-x_{12} x_{5}^{\prime}-x_{13} x_{4}^{\prime}-x_{14} x_{3}^{\prime}+x_{15} x_{2}^{\prime}+x_{16} x_{1}^{\prime}\right) \\
& +\omega^{2} w_{6}\left(x_{1} x_{16}^{\prime}-x_{2} x_{15}^{\prime}+x_{3} x_{14}^{\prime}-x_{4} x_{13}^{\prime}+x_{5} x_{12}^{\prime}-x_{6} x_{11}^{\prime}+x_{7} x_{10}^{\prime}-x_{8} x_{9}^{\prime}\right. \\
& \left.-x_{9} x_{8}^{\prime}+x_{10} x_{7}^{\prime}-x_{11} x_{6}^{\prime}+x_{12} x_{5}^{\prime}-x_{13} x_{4}^{\prime}+x_{14} x_{3}^{\prime}-x_{15} x_{2}^{\prime}+x_{16} x_{1}^{\prime}\right)
\end{aligned}
$$

For our purposes, it suffices to have the $16 \times 16 \times 16 \times 16$ and $3 \times 3 \times 3 \times 3$ invariants for the case where all four fields are the same.

\section{$(\mathrm{xv}) 16 \times 16 \times 16 \times 16$ invariants $\left(x_{i} \sim 16\right)$ :}

$$
\begin{aligned}
h_{1}\left(x_{i}\right) & =x_{1}^{2} x_{16}^{2}+x_{2}^{2} x_{15}^{2}+x_{3}^{2} x_{14}^{2}+x_{4}^{2} x_{13}^{2}+x_{5}^{2} x_{12}^{2}+x_{6}^{2} x_{11}^{2}+x_{7}^{2} x_{10}^{2}+x_{8}^{2} x_{9}^{2}, \\
h_{2}\left(x_{i}\right) & =x_{1} x_{2} x_{15} x_{16}+x_{1} x_{3} x_{14} x_{16}+x_{2} x_{4} x_{13} x_{15}+x_{3} x_{4} x_{13} x_{14}+x_{1} x_{5} x_{12} x_{16}+x_{4} x_{5} x_{12} x_{13} \\
& +x_{2} x_{6} x_{11} x_{15}+x_{3} x_{6} x_{11} x_{14}+x_{5} x_{6} x_{11} x_{12}+x_{2} x_{7} x_{10} x_{15}+x_{3} x_{7} x_{10} x_{14}+x_{5} x_{7} x_{10} x_{12} \\
& +x_{1} x_{8} x_{9} x_{16}+x_{4} x_{8} x_{9} x_{13}+x_{6} x_{8} x_{9} x_{11}+x_{7} x_{8} x_{9} x_{10}
\end{aligned}
$$

(xvi) $3 \times 3 \times 3 \times 3$ invariants $\left(w_{i} \sim 3\right)$ :

$$
\begin{aligned}
& h_{3}\left(w_{i}\right)=w_{1}^{4}+w_{2}^{4}+w_{3}^{4}, \\
& h_{4}\left(w_{i}\right)=w_{1}^{2} w_{2}^{2}+w_{1}^{2} w_{3}^{2}+w_{2}^{2} w_{3}^{2},
\end{aligned}
$$

\section{Appendix C}

In this appendix, we show how the light charged lepton mass matrix is computed. In the basis with $\left(\bar{e}_{L 1}, \bar{e}_{L 2}, \bar{e}_{L 3}, \bar{E}_{L 1}, \bar{E}_{L 2}, \bar{E}_{L 3}\right)$ on the left and $\left(e_{R 1}, e_{R 2}, e_{R 3}, E_{R 1}, E_{R 2}, E_{R 3}\right)$ on the right, the mass matrix has the form

$$
\mathcal{M}_{e}=\left(\begin{array}{cc}
0 & M^{\prime} \\
m & M
\end{array}\right)
$$


with

$$
\begin{gathered}
m=\left(\begin{array}{ccc}
\kappa v & 0 & 0 \\
0 & \kappa v & 0 \\
0 & 0 & \kappa v
\end{array}\right), \\
M=\left(\begin{array}{ccc}
m_{E} & 0 & 0 \\
0 & m_{E} & 0 \\
0 & 0 & m_{E}
\end{array}\right),
\end{gathered}
$$

and

$$
M^{\prime}=\left(\begin{array}{ccc}
\epsilon_{1} v_{\chi} & \epsilon_{1} v_{\chi} & \epsilon_{1} v_{\chi} \\
\epsilon_{2} v_{\chi} & \omega^{2} \epsilon_{2} v_{\chi} & \omega \epsilon_{2} v_{\chi} \\
\epsilon_{3} v_{\chi} & \omega \epsilon_{3} v_{\chi} & \omega^{2} \epsilon_{3} v_{\chi}
\end{array}\right)=\left(\begin{array}{ccc}
\epsilon_{1} & 0 & 0 \\
0 & \epsilon_{2} & 0 \\
0 & 0 & \epsilon_{3}
\end{array}\right)\left(\begin{array}{ccc}
1 & 1 & 1 \\
1 & \omega^{2} & \omega \\
1 & \omega & \omega^{2}
\end{array}\right)
$$

Here, $m$ only contains entries at the EW scale, while $M$ and $M^{\prime}$ contain entries at the higher scale $M_{*}$. To order $M_{W}^{2} / M_{*}^{2}$, the left-handed mass-squared matrix $\mathcal{M}_{e}^{\dagger} \mathcal{M}_{e}$ is block-diagonalized by [18]

$$
\mathcal{U}_{L}=\left(\begin{array}{cc}
I & m^{\dagger} M\left(M^{\dagger} M+M^{\prime \dagger} M^{\prime}\right)^{-1} \\
\left(M^{\dagger} M+M^{\prime \dagger} M^{\prime}\right)^{-1} M^{\dagger} m & I
\end{array}\right) .
$$

The upper left entry of $\mathcal{U}_{L} \mathcal{M}_{e}^{\dagger} \mathcal{M}_{e} \mathcal{U}_{L}^{\dagger}$ is the light left-handed mass-squared matrix

$$
\begin{aligned}
& M_{e}^{\dagger} M_{e}=m^{\dagger} m-m^{\dagger} M\left(M^{\dagger} M+M^{\prime \dagger} M^{\prime}\right)^{-1} M^{\dagger} m
\end{aligned}
$$

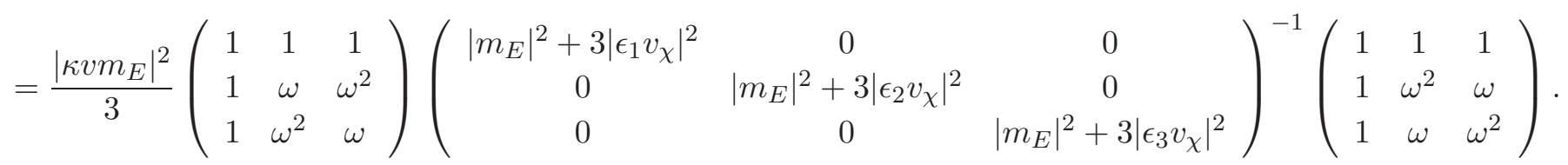

This yields the masses given in (18).

\section{Appendix D}

In this appendix, we show how the neutrino mass matrix is computed. From Appendix B, the term in Eq. (10) that mixes $N$ and $N^{\prime}$ is

$$
\begin{aligned}
g_{3}\left(N,\langle\phi\rangle, N^{\prime}\right) & =v_{\phi} N_{1}\left(N_{35}^{\prime}+N_{36}^{\prime}+N_{39}^{\prime}+N_{40}^{\prime}+N_{41}^{\prime}+N_{42}^{\prime}+N_{45}^{\prime}+N_{46}^{\prime}\right) \\
& +v_{\phi} N_{2}\left(N_{5}^{\prime}+N_{6}^{\prime}+N_{7}^{\prime}+N_{8}^{\prime}+N_{9}^{\prime}+N_{10}^{\prime}+N_{11}^{\prime}+N_{12}^{\prime}\right) \\
& +v_{\phi} N_{3}\left(N_{19}^{\prime}+N_{20}^{\prime}+N_{21}^{\prime}+N_{22}^{\prime}+N_{27}^{\prime}+N_{28}^{\prime}+N_{29}^{\prime}+N_{30}^{\prime}\right) .
\end{aligned}
$$


The term that mixes $N^{\prime}$ and $N^{\prime \prime}$ is

$$
\begin{aligned}
g_{4}\left(N^{\prime \prime},\langle\phi\rangle, N^{\prime}\right) & =v_{\phi} N_{1}^{\prime \prime}\left(N_{5}^{\prime}+N_{7}^{\prime}+N_{9}^{\prime}+N_{11}^{\prime}\right)+v_{\phi} N_{2}^{\prime \prime}\left(N_{6}^{\prime}+N_{8}^{\prime}+N_{10}^{\prime}+N_{12}^{\prime}\right) \\
& +v_{\phi} N_{3}^{\prime \prime}\left(N_{5}^{\prime}+N_{6}^{\prime}+N_{9}^{\prime}+N_{10}^{\prime}\right)+v_{\phi} N_{4}^{\prime \prime}\left(N_{7}^{\prime}+N_{8}^{\prime}+N_{11}^{\prime}+N_{12}^{\prime}\right) \\
& -v_{\phi} N_{5}^{\prime \prime}\left(N_{1}^{\prime}+N_{2}^{\prime}+N_{3}^{\prime}+N_{4}^{\prime}\right)-v_{\phi} N_{6}^{\prime \prime}\left(N_{13}^{\prime}+N_{14}^{\prime}+N_{15}^{\prime}+N_{16}^{\prime}\right) \\
& -v_{\phi} N_{7}^{\prime \prime}\left(N_{1}^{\prime}+N_{2}^{\prime}+N_{3}^{\prime}+N_{4}^{\prime}\right)-v_{\phi} N_{8}^{\prime \prime}\left(N_{13}^{\prime}+N_{14}^{\prime}+N_{15}^{\prime}+N_{16}^{\prime}\right) \\
& +v_{\phi} N_{1}^{\prime \prime}\left(N_{19}^{\prime}+N_{21}^{\prime}+N_{27}^{\prime}+N_{29}^{\prime}\right)+v_{\phi} N_{2}^{\prime \prime}\left(N_{20}^{\prime}+N_{22}^{\prime}+N_{28}^{\prime}+N_{30}^{\prime}\right) \\
& -v_{\phi} N_{3}^{\prime \prime}\left(N_{19}^{\prime}+N_{20}^{\prime}+N_{21}^{\prime}+N_{22}^{\prime}\right)-v_{\phi} N_{4}^{\prime \prime}\left(N_{27}^{\prime}+N_{28}^{\prime}+N_{29}^{\prime}+N_{30}^{\prime}\right) \\
& +v_{\phi} N_{5}^{\prime \prime}\left(N_{17}^{\prime}+N_{18}^{\prime}+N_{25}^{\prime}+N_{26}^{\prime}\right)+v_{\phi} N_{6}^{\prime \prime}\left(N_{23}^{\prime}+N_{29}^{\prime}+N_{31}^{\prime}+N_{32}^{\prime}\right) \\
& -v_{\phi} N_{7}^{\prime \prime}\left(N_{17}^{\prime}+N_{18}^{\prime}+N_{25}^{\prime}+N_{26}^{\prime}\right)-v_{\phi} N_{8}^{\prime \prime}\left(N_{23}^{\prime}+N_{29}^{\prime}+N_{31}^{\prime}+N_{32}^{\prime}\right) \\
& +v_{\phi} N_{1}^{\prime \prime}\left(N_{35}^{\prime}+N_{39}^{\prime}+N_{41}^{\prime}+N_{45}^{\prime}\right)+v_{\phi} N_{2}^{\prime \prime}\left(N_{36}^{\prime}+N_{40}^{\prime}+N_{42}^{\prime}+N_{46}^{\prime}\right) \\
& -v_{\phi} N_{3}^{\prime \prime}\left(N_{35}^{\prime}+N_{36}^{\prime}+N_{41}^{\prime}+N_{42}^{\prime}\right)-v_{\phi} N_{4}^{\prime \prime}\left(N_{39}^{\prime}+N_{40}^{\prime}+N_{45}^{\prime}+N_{46}^{\prime}\right) \\
& +v_{\phi} N_{5}^{\prime \prime}\left(N_{33}^{\prime}+N_{34}^{\prime}+N_{37}^{\prime}+N_{38}^{\prime}\right)+v_{\phi} N_{6}^{\prime \prime}\left(N_{43}^{\prime}+N_{44}^{\prime}+N_{47}^{\prime}+N_{48}^{\prime}\right) \\
& -v_{\phi} N_{7}^{\prime \prime}\left(N_{33}^{\prime}+N_{34}^{\prime}+N_{37}^{\prime}+N_{38}^{\prime}\right)-v_{\phi} N_{8}^{\prime \prime}\left(N_{43}^{\prime}+N_{44}^{\prime}+N_{47}^{\prime}+N_{48}^{\prime}\right) .
\end{aligned}
$$

Since the symmetries $B_{1}, B_{2}, B_{3} B_{4}$, and $A_{3} A_{4}$ are unbroken, components of $N^{\prime}$ and $N^{\prime \prime}$ that transform under these symmetries cannot mix with the light neutrinos. This leaves

$$
\begin{aligned}
& p_{1}=\frac{N_{5}^{\prime}+N_{6}^{\prime}+N_{7}^{\prime}+N_{8}^{\prime}+N_{9}^{\prime}+N_{10}^{\prime}+N_{11}^{\prime}+N_{12}^{\prime}}{\sqrt{8}}, \\
& p_{2}=\frac{N_{19}^{\prime}+N_{20}^{\prime}+N_{21}^{\prime}+N_{22}^{\prime}+N_{27}^{\prime}+N_{28}^{\prime}+N_{29}^{\prime}+N_{30}^{\prime}}{\sqrt{8}}, \\
& p_{3}=\frac{N_{35}^{\prime}+N_{36}^{\prime}+N_{39}^{\prime}+N_{40}^{\prime}+N_{41}^{\prime}+N_{42}^{\prime}+N_{45}^{\prime}+N_{46}^{\prime}}{\sqrt{8}}, \\
& q_{1}=\frac{N_{1}^{\prime \prime}+N_{2}^{\prime \prime}}{\sqrt{2}}, \quad q_{2}=\frac{N_{3}^{\prime \prime}+N_{4}^{\prime \prime}}{\sqrt{2}} .
\end{aligned}
$$

We now have

$$
\begin{aligned}
g_{3}\left(N,\langle\phi\rangle, N^{\prime}\right) & =\sqrt{8} v_{\phi}\left(N_{1} p_{3}+N_{2} p_{1}+N_{3} p_{2}\right), \\
g_{4}\left(N^{\prime \prime},\langle\phi\rangle, N^{\prime}\right) & =2 v_{\phi}\left(q_{1} p_{1}+q_{2} p_{1}+q_{1} p_{2}-q_{2} p_{2}+q_{1} p_{3}-q_{2} p_{3}\right)+\ldots,
\end{aligned}
$$

where the ellipses in the second equation refer to terms involving only decoupled components. The mass matrix for $\left(\nu_{1}, \nu_{2}, \nu_{3}, N_{1}, N_{2}, N_{3}, p_{1}, p_{2}, p_{3}, q_{1}, q_{2}\right)$ has the form

$$
\mathcal{M}_{\nu}=\left(\begin{array}{cc}
0 & m \\
m^{T} & M
\end{array}\right)
$$

with

$$
m=\left(\begin{array}{cccccccc}
\frac{1}{2} \lambda v & 0 & 0 & 0 & 0 & 0 & 0 & 0 \\
0 & \frac{1}{2} \lambda v & 0 & 0 & 0 & 0 & 0 & 0 \\
0 & 0 & \frac{1}{2} \lambda v & 0 & 0 & 0 & 0 & 0
\end{array}\right)
$$




$$
M=\left(\begin{array}{cccccccc}
m_{N} & 0 & 0 & 0 & 0 & \sqrt{2} \alpha_{1} v_{\phi} & 0 & 0 \\
0 & m_{N} & 0 & \sqrt{2} \alpha_{1} v_{\phi} & 0 & 0 & 0 & 0 \\
0 & 0 & m_{N} & 0 & \sqrt{2} \alpha_{1} v_{\phi} & 0 & 0 & 0 \\
0 & \sqrt{2} \alpha_{1} v_{\phi} & 0 & m_{N}^{\prime}+\beta v_{\phi} & 0 & 0 & \alpha_{2} v_{\phi} & \alpha_{2} v_{\phi} \\
0 & 0 & \sqrt{2} \alpha_{1} v_{\phi} & 0 & m_{N}^{\prime}+\beta v_{\phi} & 0 & \alpha_{2} v_{\phi} & -\alpha_{2} v_{\phi} \\
\sqrt{2} \alpha_{1} v_{\phi} & 0 & 0 & 0 & 0 & m_{N}^{\prime}+\beta v_{\phi} & \alpha_{2} v_{\phi} & -\alpha_{2} v_{\phi} \\
0 & 0 & 0 & \alpha_{2} v_{\phi} & \alpha_{2} v_{\phi} & \alpha_{2} v_{\phi} & m_{N}^{\prime \prime} & 0 \\
0 & 0 & 0 & \alpha_{2} v_{\phi} & -\alpha_{2} v_{\phi} & -\alpha_{2} v_{\phi} & 0 & m_{N}^{\prime \prime}
\end{array}\right) .
$$

Here, $m$ only contains entries at the EW scale, while $M$ contains entries at the higher scale $M_{*}$. To order $M_{W}^{2} / M_{*}^{2}, \mathcal{M}_{\nu}$ is block-diagonalized by

$$
\mathcal{U}_{\nu}=\left(\begin{array}{cc}
I & -m M^{-1} \\
M^{-1} m^{T} & I
\end{array}\right) .
$$

The light neutrino mass matrix $M_{\nu}$ is given by the upper-left block of $\mathcal{U}_{\nu} \mathcal{M}_{\nu} \mathcal{U}_{\nu}^{T}$,

$$
M_{\nu}=-m M^{-1} m^{T}
$$

Let

$$
S=\frac{1}{\sqrt{2}}\left(\begin{array}{cccccccc}
1 & 0 & 0 & 0 & 0 & 0 & 1 & 0 \\
0 & 0 & 0 & \sqrt{2} & 0 & 0 & 0 & 0 \\
1 & 0 & 0 & 0 & 0 & 0 & -1 & 0 \\
0 & 0 & 0 & 0 & \sqrt{2} & 0 & 0 & 0 \\
0 & 1 & 0 & 0 & 0 & 0 & 0 & 1 \\
0 & 1 & 0 & 0 & 0 & 0 & 0 & -1 \\
0 & 0 & 1 & 0 & 0 & 1 & 0 & 0 \\
0 & 0 & -1 & 0 & 0 & 1 & 0 & 0
\end{array}\right)
$$

Then,

$$
S^{-1} M S=\left(\begin{array}{ccc}
A & 0 & 0 \\
0 & B & 0 \\
0 & 0 & C
\end{array}\right)
$$

with

$$
\begin{gathered}
A=\left(\begin{array}{ccc}
m_{N} & \sqrt{2} \alpha_{1} v_{\phi} & 0 \\
\sqrt{2} \alpha_{1} v_{\phi} & m_{N}^{\prime}+\beta v_{\phi} & 2 \alpha_{2} v_{\phi} \\
0 & 2 \alpha_{2} v_{\phi} & m_{N}^{\prime \prime}
\end{array}\right), \\
B=\left(\begin{array}{ccc}
m_{N} & \sqrt{2} \alpha_{1} v_{\phi} & 0 \\
\sqrt{2} \alpha_{1} v_{\phi} & m_{N}^{\prime}+\beta v_{\phi} & \sqrt{2} \alpha_{2} v_{\phi} \\
0 & \sqrt{2} \alpha_{2} v_{\phi} & m_{N}^{\prime \prime}
\end{array}\right),
\end{gathered}
$$




$$
C=\left(\begin{array}{cc}
m_{N} & -\sqrt{2} \alpha_{1} v_{\phi} \\
-\sqrt{2} \alpha_{1} v_{\phi} & m_{N}^{\prime}+\beta v_{\phi}
\end{array}\right)
$$

So, we can write

$$
\begin{gathered}
M_{\nu}=-m S\left(\begin{array}{ccc}
A^{-1} & 0 & 0 \\
0 & B^{-1} & 0 \\
0 & 0 & C^{-1}
\end{array}\right) S^{-1} m^{T} \\
=-\frac{\lambda^{2} v^{2}}{8}\left(\begin{array}{ccc}
\left(A^{-1}\right)_{11}+\left(C^{-1}\right)_{11} & 0 & \left(A^{-1}\right)_{11}-\left(C^{-1}\right)_{11} \\
0 & 2\left(B^{-1}\right)_{11} & 0 \\
\left(A^{-1}\right)_{11}-\left(C^{-1}\right)_{11} & 0 & \left(A^{-1}\right)_{11}+\left(C^{-1}\right)_{11}
\end{array}\right) .
\end{gathered}
$$

This mass matrix is diagonalized by (20), and the masses are given by

$$
m_{1}=\left|\frac{\lambda^{2} v^{2}}{4}\left(A^{-1}\right)_{11}\right|, \quad m_{2}=\left|\frac{\lambda^{2} v^{2}}{4}\left(B^{-1}\right)_{11}\right|, \quad m_{3}=\left|\frac{\lambda^{2} v^{2}}{4}\left(C^{-1}\right)_{11}\right|
$$

\section{Acknowledgments}

This work is supported in part by Department of Energy Grant Numbers DE-FG02-04ER41306 and DE-FG02-ER46140.

\section{References}

[1] T. Schwetz, M. Tortola, and J.W.F. Valle, New J. Phys. 10, 113011 (2008).

[2] G.L. Fogli, E. Lisi, A. Mirizzi, D. Montanino, and P.D. Serpico, Phys. Rev. D74, 093004 (2006).

[3] P.F. Harrison, D.H. Perkins and W.G. Scott, Phys. Lett. B458, 79 (1999); Phys. Lett. B530, 167 (2002).

[4] Z.-Z. Xing, Phys. Lett. B533, 85 (2002);

X.G. He and A. Zee, Phys. Lett. B560, 87 (2003); Phys. Rev. D68, 037302 (2003).

[5] E. Ma and G. Rajasekaran, Phys. Rev. D64, 113012 (2001).

[6] E. Ma, Mod. Phys. Lett. A17, 2361 (2002).

[7] K.S. Babu, E. Ma, and J.W.F. Valle, Phys. Lett. B552, 207 (2003).

[8] G. Altarelli and F. Feruglio, Nucl. Phys. B720, 64 (2005); Nucl. Phys. B741, 215 (2006). 
[9] K. S. Babu and X.G. He, hep-ph/0507217.

[10] X.G. He, Y.Y. Keum, and R.R. Volkas, JHEP 0604, 039 (2006).

[11] E. Ma, Phys. Lett. B671, 366 (2009).

[12] M. Hirsch, S. Morisi and J. W. F. Valle, Phys. Rev. D 79, 016001 (2009).

[13] F. Feruglio, C. Hagedorn and L. Merlo, JHEP 1003, 084 (2010).

[14] For recent reviews see: E. Ma, arXiv:0705.0327 [hep-ph];

G. Altarelli and F. Feruglio, arXiv:1002.0211 [hep-ph];

H. Ishimori, T. Kobayashi, H. Ohki, H. Okada, Y. Shimizu and M. Tanimoto, arXiv:1003.3552 [hep-th].

[15] C. Hagedorn, M. Lindner and R. N. Mohapatra, JHEP 0606, 042 (2006);

M. C. Chen and K. T. Mahanthappa, Phys. Lett. B 652, 34 (2007);

C. Luhn, S. Nasri and P. Ramond, J. Math. Phys. 48, 123519 (2007);

I. de Medeiros Varzielas, S. F. King and G. G. Ross, Phys. Lett. B 648, 201 (2007);

W. Grimus and L. Lavoura, JHEP 0904, 013 (2009); JHEP 0809, 106 (2008);

E. Ma, Phys. Lett. B 660, 505 (2008);

L. L. Everett and A. J. Stuart, Phys. Rev. D 79, 085005 (2009);

P. H. Frampton and S. Matsuzaki, Phys. Lett. B 679, 347 (2009);

B. Adhikary, A. Ghosal and P. Roy, JHEP 0910, 040 (2009);

H. Ishimori, K. Saga, Y. Shimizu and M. Tanimoto, arXiv:1004.5004 [hep-ph];

C. Hagedorn, S. F. King and C. Luhn, arXiv:1003.4249 [hep-ph].

[16] S.K. Kim, Group Theoretical Methods: And Applications to Molecules and Crystals, Cambridge University Press, New York, 1999.

[17] C.S. Lam, Phys. Rev. D79, 073015 (2008);

W. Grimus, L. Lavoura, and P.O. Ludl, 0906.2689 [hep-ph].

[18] See for e.g., K. S. Babu and S. M. Barr, Phys. Rev. D 56, 2614 (1997). 